\title{
On ice-induced instability in free-surface flows
}

\author{
EVGENIY SHAPIRO ${ }^{1}$ AND SERGEI TIMOSHIN ${ }^{2}$ \\ ${ }^{1}$ Department of Aerospace Sciences, Cranfield University, Cranfield MK43 0AL, UK \\ ${ }^{2}$ Department of Mathematics, University College London, Gower Street, London WC1 6BT, UK
}

(Received 8 November 2005 and in revised form 17 October 2006)

The problem of stability of a water-coated ice layer is investigated for a free-surface flow of a thin water film down an inclined plane. An asymptotic (double-deck) theory is developed for a flow with large Reynolds and Froude numbers which is then used to investigate linear two-dimensional, three-dimensional and nonlinear two-dimensional stability characteristics. A new mode of upstream-propagating instability arising from the interaction of the ice surface with the flow is discovered and its properties are investigated. In the linear limit, closed-form expressions for the dispersion relation and neutral curves are obtained for the case of $\operatorname{Pr}=1$. For the general case, the linear stability problem is solved numerically and the applicability of the solution with $\operatorname{Pr}=1$ is analysed. Nonlinear double-deck equations are solved with a novel globalmarching-type scheme and the effects of nonlinearity are investigated. An explanation of the physical mechanism leading to the upstream propagation of instability waves is provided.

\section{Introduction}

The problem of solidification front stability arises in a number of engineering applications including casting (Glicksman, Coriell \& McFadden 1986), pipe freezing (Gilpin 1979, 1981; Seki, Fukusako \& Younan 1984) and airplane icing (Vargas \& Reshotko 1998; Rothmayer \& Tsao 1998). In all these applications we encounter an interaction between an unsteady solidification front and the flow of the liquid phase which can lead to instability of the solidification front.

Hydrodynamic and morphological instabilities of the solidification interface in alloy solidification and crystal growth have been the subject of extensive research (see, for example, Glicksman et al. 1986; Davis 1990; Batchelor, Moffatt \& Worster 2000). As a result, the coupling of a forced flow with morphological instabilities is a wellknown effect in the models where the interface formation is determined by the solute concentration field near the solid/liquid interface. A characteristic feature of the solidification front instability observed in these problems is the upstream propagation of the solid/liquid interface disturbance (see Brattkus \& Davis 1988; Forth \& Wheeler 1989).

The question of stability of the temperature-driven solidification fronts, and icing in particular, has received comparatively little attention despite the experimental evidence available. Gilpin (1979) performed a series of experiments in order to study ice growth in a pipe at or near Reynolds numbers typical of laminar-turbulent transition. He found that for the entire range of Reynolds numbers that was obtainable in the experiment (up to 14000), there existed a final steady-state ice profile with a cyclic variation in height along the length of the pipe - an 'ice-band' structure. However, another similar experiment by Kikuchi et al. (1986) demonstrated a smooth 
steady-state ice surface development without the ice-band structures. Gilpin (1981) extended the earlier study to the range of transitional and turbulent flows. In this second paper, he attributed the upstream propagation to the turbulent heat transfer downstream of the separation point (separation occurred on the downstream inclined ice slope). This explanation, however, does not seem to account for the initial stages of the ice-band formation, when the perturbation of the uniform ice surface is small, and no flow separation occurs. Seki et al. (1984) studied ice formation experimentally in a water flow between two cooled parallel plates. They found that, depending on the experimental conditions, expansions can form in the melt passageway and migrate upstream, as in the experiments reported by Gilpin $(1979,1981)$. Similar behaviour with characteristic upstream propagation was reported for ice formation under a water film running down an inclined plane by Streitz \& Ettema (2002).

Yao \& Prusa (1989) in their review paper on solidification and melting noted that the phenomenon of the solidification front instability has been extensively observed experimentally, but has been almost completely ignored in modelling.

One attempt was made recently within the context of aircraft icing modelling. The question of the influence of an ice layer on the flow stability for a boundary-layer-scale water film was tackled analytically by Rothmayer \& Tsao (1998). They developed a two-dimensional viscous-inviscid interaction triple-deck theory in order to describe the interaction between the air boundary layer, water film and ice sheet. This tripledeck theory was further developed in Rothmayer \& Tsao (2000). As a result of their analysis, a new broadband ice instability mode was discovered in regimes with simultaneous air and water cooling. However, the interpretation of the results in terms of flow stability proves difficult, since there is no steady or base-state solution for the flow in the problem investigated. Another difficulty of the complete triple-deck approach is the number of instability mechanisms present in the problem and their interaction, see, for example Timoshin (1997) and Timoshin \& Hooper (2000).

In this paper we consider a gravity-driven water film flow on an inclined plane in order to understand how the interaction between the ice layer and the flow affects the stability characteristics. The film is considered at a boundary-layer scale in order to approach aircraft icing conditions. We investigate this problem in the limit of large Froude numbers and derive a double-deck theory, which allows us to isolate icing instability. In this approximation we investigate the linear stability of the steady-state solution, for which we obtain an analytic expression for the dispersion relation in the case of $\operatorname{Pr}=1$ and a numerical solution in the general case for both three-dimensional and two-dimensional flows. In particular, we show that icing instability emerges in a two-dimensional flow provided the ice layer is sufficiently thick (in certain cases, however, it is oblique-wave disturbances that are found to be most unstable). We also solve the fully nonlinear problem in the viscous sublayer numerically in the twodimensional case and analyse the effects of nonlinearity. Finally, based on the results obtained, we suggest a simple physical explanation of the upstream propagation effect observed in such flows.

\section{Problem formulation and the double-deck leading-order asymptotic expansion}

\subsection{Boundary-layer-scale problem}

We consider a thin water film with the same relative thickness as the boundary layer thickness in the flow. The air/water boundary is taken to be a free surface with a prescribed constant air temperature above the freezing point (i.e. warm air). From 


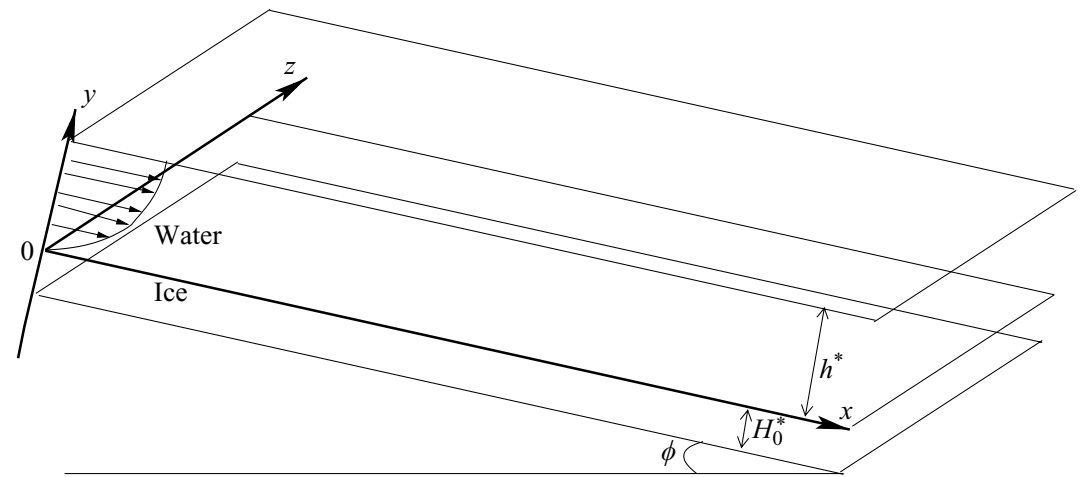

FIGURE 1. Flow scheme for a water film in the boundary-layer regime.

below, the water layer is bounded by a thin ice sheet. The ice sheet rests on a solid wall whose temperature is prescribed. The wall is inclined at an angle $\phi$ to the horizontal. The flow scheme for this configuration is shown on figure 1.

We use superscript * in order to denote variables with dimensions. For the problem under consideration it is convenient to define dimensionless pressure as

$$
P=\frac{P^{*}-P_{a}^{*}}{\rho_{l}^{*} g^{*} h^{*}}
$$

where $P^{*}$ and $P_{a}^{*}$ denote local pressure and reference (air) pressure respectively, $\rho_{l}^{*}$ is the density of the liquid, $g^{*}$ is the acceleration due to gravity and $h^{*}$ is the height of the unperturbed film. Also we will use the height of the unperturbed film $h^{*}$ as a characteristic length in the direction normal to the wall $(y)$ and assume a separate characteristic length $L^{*}$ in the streamwise and spanwise directions ( $x$ and $z$ ). We define characteristic velocity $U^{*}$ to be the integral mean velocity in the unperturbed flow. Dimensionless temperature is defined by

$$
T=\frac{T^{*}-T_{f}^{*}}{D T}
$$

where $T^{*}$ is local temperature and $D T=T_{a}^{*}-T_{f}^{*}$. $T_{f}^{*}$ and $T_{a}^{*}$ denote freezing temperature and external air temperature respectively. We denote the characteristic time of the ice growth by $\tau_{i}^{*}$. In general, this characteristic time can be different from the flow time scale

$$
\tau_{\text {flow }}^{*}=\frac{L^{*}}{U^{*}} .
$$

Dimensionless parameters are defined as follows,

$$
S r=\frac{\tau_{i}^{*}}{\tau_{\text {flow }}^{*}}=\frac{\tau_{i}^{*} U^{*}}{L^{*}}
$$

is the Strouhal number;

$$
S t=\frac{C p_{l} D T}{H_{l i}}
$$

is the Stefan number, where $C p_{l}$ and $H_{l i}$ denote the specific heat of water and the latent heat of ice/water phase change respectively;

$$
\Theta_{i l}=1-\frac{\rho_{i}}{\rho_{l}}
$$


is the volume change parameter depending on the ratio of ice density $\rho_{i}$ to water density $\rho_{l}$;

$$
R e=\frac{U^{*} L^{*}}{v_{l}^{*}}
$$

is the Reynolds number;

$$
\operatorname{Pr}=\frac{v_{l}^{*}}{\kappa_{l}^{*}}
$$

is the Prandtl number where $v_{l}^{*}$ and $\kappa_{l}^{*}$ denote kinematic viscosity and thermal diffusivity of water respectively;

$$
F r=\frac{U^{* 2}}{g^{*} h^{*}}
$$

denotes the Froude number;

$$
W e=\frac{\rho_{l}^{*} U^{* 2} L^{*}}{\gamma^{*}}
$$

is the Weber, where $\gamma^{*}$ is the surface tension coefficient.

We will assume that the film thickness is of the same order of magnitude as the thickness of the boundary layer and $h^{*} / L^{*}=R e^{-0.5} \ll 1$. Note that the approach can be generalized by taking $\sqrt{R e}=C L^{*} / h^{*}$ where $C \sim 1$ is a constant, which will result in the multiplication of viscous terms by a $1 / C^{2}$ coefficient. However the addition of this coefficient will increase the parametric space of the problem and will not lead to a qualitative difference in the results; therefore we restrict our discussion to the case of $C=1$.

In order to isolate the icing instability, we consider the problem on the time scale of the evolution of the ice sheet. The evolution of the ice surface is linked to the flow field via the Stefan condition (see, for example Alexiades \& Solomon 1993). We are looking for the characteristic time at which the Stefan condition remains in its full form. The Stefan condition written in dimensionless form in boundary-layer scaling yields

$$
\lambda_{i l} \frac{\partial T_{i}}{\partial \boldsymbol{n}}-\frac{\partial T}{\partial \boldsymbol{n}}=\Theta_{i l} \frac{1}{S t S r} \operatorname{Pr} \frac{1}{|\nabla H|} \frac{\partial H}{\partial t},
$$

where $T_{i}$ denotes ice temperature, $H(x, y, z, t)=0$ is an implicit equation of the ice surface and $\boldsymbol{n}$ is a unit vector normal to the ice surface. In order to preserve the interaction between the ice layer and the flow, it is necessary to maintain the full form of the Stefan condition (2.1). Then the dimensionless combination on the right-hand side of (2.1) should be $O(1)$, which yields the following estimate for the Strouhal number:

$$
S r \sim \frac{\Theta_{i l} P r}{S t} .
$$

For water near $0^{\circ} \mathrm{C}$ and $D T \sim O(1)$, the Stefan number based on water properties is $O\left(10^{-2}\right)$ and $\operatorname{Pr} \Theta_{i l} \sim O(1)$. Then in order to preserve Stefan condition in the boundary-layer scaling

$$
S r \sim \frac{1}{S t},
$$

from which we can obtain the characteristic time of ice growth

$$
\tau_{i} \sim \frac{L^{*}}{S t U^{*}} .
$$

This simple qualitative analysis also shows that the time required for the ice structure to reach a final steady state in a temporal evolution should be inversely proportional 
to the Stefan number. This was observed in numerical computations of ice growth in a pipe flow by Lee (1993). Note that under these conditions $S r \gg 1$ and the time derivatives can be neglected in the boundary layer equations.

Using the characteristic time defined by (2.4) and assuming that the Reynolds number is large we obtain the following initial system of dimensionless equations in the water layer:

$$
\begin{gathered}
\frac{\partial u}{\partial x}+\frac{\partial v}{\partial y}+\frac{\partial w}{\partial z}=0 \\
u \frac{\partial u}{\partial x}+v \frac{\partial u}{\partial y}+w \frac{\partial u}{\partial z}=-\frac{1}{F r} \frac{\partial P}{\partial x}+\frac{\sqrt{\operatorname{Re}} \sin \phi}{F r}+\frac{\partial^{2} u}{\partial y^{2}} \\
\frac{\partial P}{\partial y}=-\cos \phi \\
u \frac{\partial w}{\partial x}+v \frac{\partial w}{\partial y}+w \frac{\partial w}{\partial z}=-\frac{1}{F r} \frac{\partial P}{\partial z}+\frac{\partial^{2} w}{\partial y^{2}}, \\
u \frac{\partial T}{\partial x}+v \frac{\partial T}{\partial y}+w \frac{\partial T}{\partial z}=\frac{1}{P r} \frac{\partial^{2} T}{\partial y^{2}},
\end{gathered}
$$

as is usual for a boundary-layer-type flow, with $u, v$ and $w$ denoting the components of dimensionless velocity in directions $x, y$ and $z$ respectively. The heat conduction in the ice layer is governed by the reduced heat equation for the ice temperature

$$
\frac{\partial^{2} T_{i}}{\partial y^{2}}=0
$$

on account of the assumed small thickness of the ice sheet and the relatively large time scale of the ice development.

On the upper boundary of the water layer we assume constant air pressure and zero friction. Then the boundary conditions at the air/water interface $(y=f(x, z, t))$ are given by

$$
\begin{gathered}
v-u \frac{\partial f}{\partial x}-w \frac{\partial f}{\partial z}=0, \\
\frac{\partial u}{\partial y}=0, \\
\frac{\partial w}{\partial y}=0, \\
P=-\frac{\sin \phi}{3 W e}\left(\frac{\partial^{2} f}{\partial x^{2}}+\frac{\partial^{2} f}{\partial z^{2}}\right), \\
T=1,
\end{gathered}
$$

Equation $(2.7 a)$ corresponds to the kinematic condition, $(2.7 b-d)$ are derived from the zero stress condition and (2.7e) stipulates constant air temperature. At the water/ice interface $y=h(x, z, t)$, we obtain

$$
\left.\begin{array}{c}
u=v=w=0, \\
T=T_{i}=0, \\
\frac{\partial T_{i}}{\partial y}-\frac{\partial T}{\partial y}=\operatorname{Pr} \Theta_{i l} \frac{\partial h}{\partial t}
\end{array}\right\}
$$

where $\lambda_{i l}=\lambda_{i} / \lambda_{l}$ is the ratio of ice thermal conductivity $\lambda_{i}$ to liquid thermal conductivity $\lambda_{l}$. Note that since we are considering a problem on the ice time scale, the time 
derivative is retained in the Stefan condition but not in the kinematic condition. The conditions for velocity in (2.8) are derived from the kinematic condition at the ice surface and the zero tangential velocity condition, taking into account boundary-layer scaling and large Strouhal number. The last two equations of (2.8) represent constant freezing temperature and the Stefan condition respectively. Finally, at the solid wall $\left(y=-H_{0}\right)$, we require a constant ice temperature,

$$
T_{i}=T_{w},
$$

where $T_{w}$ denotes the constant wall temperature.

The base flow solution to be used in the stability calculations below corresponds to flat water/ice and air/water interfaces. It is given by a half-Poiseuille velocity profile with linear temperature profiles in water and in ice:

$$
\left.\begin{array}{ll}
u_{0}=3\left(y-\frac{1}{2} y^{2}\right), & v_{0}=w_{0}=0, \\
P_{0}=(1-y) \cos \phi, & T_{0}=y, \\
T_{0 i}=-\frac{y}{H_{0}} T_{w}, &
\end{array}\right\},
$$

Also, as the solution in the undisturbed state (for example, far upstream of the region under consideration) is gravity-driven, viscous dissipation balances gravity parallel to the slope (see, for example, Bowles 1995) which leads to

$$
\frac{\sqrt{\operatorname{Re}} \sin \phi}{\operatorname{Fr}}=3 \text {. }
$$

\subsection{The limit of large Froude numbers}

In order to simplify the formulation a little further, we consider the large Froude number limit, $F r \gg 1$. Then the flow field can be divided into two layers with the lower 'viscous' layer maintaining the balance between inertia and viscous forces and the upper layer governed by predominantly inviscid dynamics. The derivation is generally similar to the analysis of the boundary-layer flows in the triple-deck theory, see, for example, Sychev et al. 2000). Of more relevance to the present study is the double-deck asymptotic theory of liquid film flows in Gajjar (1987).

Let us derive the scales for the lower viscous layer. From the form of the base flow solution (2.10), we can see that in the viscous sublayer (denoted by subscript 1 ) $u_{1} \sim y_{1}$ and in order to maintain all terms in the continuity equation, we need $v_{1} \sim y_{1}^{2} / x_{1}$ and $w_{1} \sim y_{1} z_{1} / x_{1}$. In order to maintain the full form of the $x_{1}$ momentum equation, we need

$$
\frac{1}{F r} \frac{\partial P_{1}}{\partial x_{1}} \sim \frac{\partial^{2} u_{1}}{\partial y_{1}^{2}}, \quad \text { therefore } \frac{P_{1}}{x_{1} F r} \sim \frac{1}{y_{1}}
$$

also from the balance of convective and pressure terms in the same equation

$$
\frac{P_{1}}{x_{1} F r} \sim v_{1} \frac{\partial u_{1}}{\partial y_{1}}, \quad \text { therefore } \frac{P_{1}}{x_{1} F r} \sim \frac{y_{1}^{2}}{x_{1}} .
$$

Combining these, we obtain:

$$
P_{1} \sim F r y_{1}^{2} \sim \frac{x_{1} F r}{y_{1}}
$$

therefore in the viscous sublayer $y_{1}^{3} \sim x_{1}$. Then as the variation of pressure is of the same order as the variation of height, we have $P_{1} \sim y_{1} \sim F r y_{1}^{2}$ which leads to $y_{1} \sim F r^{-1}$ and $x_{1} \sim \mathrm{Fr}^{-3}$. Similarly the balance of convective and pressure terms in the equation 
for the $z_{1}$ component of the momentum gives

$$
u_{1} \frac{\partial w_{1}}{\partial x_{1}} \sim \frac{1}{F r} \frac{\partial P_{1}}{\partial z_{1}}, \quad \text { therefore } \quad w_{1} \sim \frac{P_{1} x_{1}}{z_{1} y_{1} F r} \sim \frac{y_{1} z_{1}}{x_{1}},
$$

which leads to

$$
w_{1} \sim \frac{x_{1}}{F r z_{1}} \sim F r^{-1}
$$

Also, as the initial temperature profile is linear we have $T_{1} \sim y_{1}$ so that, in order to maintain the time derivative $\partial h / \partial t$ in the viscous sublayer, we arrive at the estimate for the time scale in the problem, $t_{1} \sim \mathrm{Fr}^{-1}$.

Based on these scaling considerations, the leading-order asymptotic expansion for the velocity, pressure and temperature in the viscous layer is given by

$$
\begin{gathered}
u=F r^{-1} u_{1}, \quad v=F r v_{1}, \quad w=F r^{-1} w_{1}, \\
P=F r^{-1} P_{1}, \quad T=F r^{-1} T_{1},
\end{gathered}
$$

which leads to the following system of equations

$$
\left.\begin{array}{c}
\frac{\partial u_{1}}{\partial x_{1}}+\frac{\partial v_{1}}{\partial y_{1}}+\frac{\partial w_{1}}{\partial z_{1}}=0, \\
u_{1} \frac{\partial u_{1}}{\partial x_{1}}+v_{1} \frac{\partial u_{1}}{\partial y_{1}}+w_{1} \frac{\partial u_{1}}{\partial z_{1}}=-\frac{\partial P_{1}}{\partial x_{1}}+\frac{\partial^{2} u_{1}}{\partial y_{1}^{2}}, \\
\frac{\partial P_{1}}{\partial y_{1}}=\cos \phi, \\
u_{1} \frac{\partial w_{1}}{\partial x_{1}}+v_{1} \frac{\partial w_{1}}{\partial y_{1}}+w_{1} \frac{\partial w_{1}}{\partial z_{1}}=-\frac{\partial P_{1}}{\partial z_{1}}+\frac{\partial^{2} w_{1}}{\partial y_{1}^{2}}, \\
u_{1} \frac{\partial T_{1}}{\partial x_{1}}+v_{1} \frac{\partial T_{1}}{\partial y_{1}}+w_{1} \frac{\partial T_{1}}{\partial z_{1}}=\frac{1}{\operatorname{Pr}} \frac{\partial^{2} T_{1}}{\partial y_{1}^{2}} .
\end{array}\right\}
$$

The ice surface height in the viscous sublayer scales with the $y$ coordinate and hence we can define $h=H_{1} \mathrm{Fr}^{-1}$, where $H_{1} \sim 1$. Then the heat transfer problem in ice can be solved readily, and we obtain the following solution for the ice temperature:

$$
T_{i}=T_{w} \frac{H_{1}-y_{1}}{H_{1}+H_{0}} .
$$

In the inviscid layer (denoted by subscript 2) we write an expansion of our solution as

$$
\begin{gathered}
u=u_{0}(y)+\epsilon u_{2}, \quad v=\epsilon v_{2}, \quad w=\epsilon w_{2}, \\
P=P_{0}(y)+\epsilon P_{2}, \quad T=T_{0}(y)+\epsilon T_{2},
\end{gathered}
$$

where the value of $\epsilon$ is obtained from scaling as follows. In the inviscid layer $x_{2} \sim x_{1} \sim F^{-3}$ and $u_{0} \sim 1$. Then, assuming that the streamline inclination is of the same order in both layers we have

$$
\frac{1}{v_{2}} \sim \frac{\sqrt{u_{1}^{2}+w_{1}^{2}}}{v_{1}} \sim F r^{-2},
$$

therefore $v_{2} \sim F r^{2}$. When we decompose the $x_{2}$ component of the velocity in the inviscid layer into a base flow part $u_{0}$ and a perturbation part $u_{2}$, from the continuity 
equation we obtain

$$
u_{2} \sim \frac{v_{2} x_{2}}{y_{2}} \sim F^{-1} .
$$

The magnitude of the pressure perturbation is the same in both layers, $P_{2} \sim P_{1} \sim F^{-1}$. Then from the balance of convective and pressure terms for the $z_{2}$ component of the momentum we obtain

$$
\frac{1}{F r} \frac{\partial P_{2}}{\partial z_{2}} \sim u_{0} \frac{\partial w_{2}}{\partial x_{2}} \text { and hence } w_{2} \sim \frac{F r^{-1} x_{2}}{z_{2} F r} .
$$

From the viscous sublayer scaling it follows that $z_{2} \sim x_{2}$ and we obtain $w_{2} \sim \mathrm{Fr}^{-2}$. Therefore in the upper inviscid layer the appropriate expansion of the solution is found in the following form (taking into account the leading order of perturbation):

$$
\begin{gathered}
u=u_{0}(y)+F r^{-1} u_{2}, \quad v=v_{2} F r^{2}, \quad w=w_{2} F r^{-2}, \\
P=P_{0}(y)+F r^{-1} P_{2}, \quad T=T_{0}(y)+F r^{-1} T_{2} .
\end{gathered}
$$

The expansion for the free-surface shape is given by $f=1+\mathrm{Fr}^{-1} F_{2}$. Then the system of equations (2.5) written in the inviscid-layer scaling becomes

$$
\left.\begin{array}{c}
u_{0} \frac{\partial u_{2}}{\partial x_{2}}+v_{2} \frac{\partial u_{0}}{\partial y_{2}}=0, \quad u_{0} \frac{\partial w_{2}}{\partial x_{2}}=-\frac{\partial P_{2}}{\partial z_{2}}, \\
\frac{\partial P_{2}}{\partial y_{2}}=0, \quad \frac{\partial u_{2}}{\partial x_{2}}+\frac{\partial v_{2}}{\partial y_{2}}=0, \\
u_{0} \frac{\partial T_{2}}{\partial x_{2}}+v_{2} \frac{\partial T_{0}}{\partial y_{2}}=0 .
\end{array}\right\}
$$

We now derive the solution in the upper inviscid layer. From the system of equations (2.14) it immediately follows that $P_{2}=P_{2}\left(x_{2}, z_{2}, t_{1}\right)$. Furthermore, from the linearized kinematic condition on the free surface, we obtain

$$
v_{2}\left(x_{2}, 1, z_{2}, t_{1}\right)=u_{0}(1) F_{2}\left(x_{2}, z_{2}, t_{1}\right) .
$$

We consider the temperature to be constant at the free surface. Expanding the boundary condition for the perturbation of temperature on the unperturbed free surface $\left(y_{2}=1\right)$ we obtain

$$
\begin{aligned}
T & =T_{0}\left(1+F r^{-1} F_{2}\right)+F r^{-1} T_{2}\left(F r^{-1} F_{2}\right) \\
& =T_{0}(1)+F r^{-1}\left(F_{2} \frac{\partial T_{0}}{\partial y_{2}}(1)+T_{2}(1)\right)+O\left(F r^{-2}\right)=T_{0}(1) .
\end{aligned}
$$

The base temperature profile $T_{0}$ satisfies the boundary condition on the unperturbed free surface, therefore

$$
T_{2}\left(x_{2}, 1, z_{2}, t_{1}\right)=-F_{2}\left(x_{2}, z_{2}, t_{1}\right) \frac{\partial T_{0}}{\partial y_{2}}(1) .
$$

From continuity of the stress tensor at the free surface we obtain the relations

$$
\frac{\partial u_{2}}{\partial y_{2}}(1)=\frac{\partial w_{2}}{\partial y_{2}}(1)=0,
$$

and also the pressure-displacement law

$$
P_{2}=F_{2} \cos \phi-\frac{\sin \phi}{3 W e}\left(\frac{\partial^{2} F_{2}}{\partial x_{2}^{2}}+\frac{\partial^{2} F_{2}}{\partial z_{2}^{2}}\right) .
$$


From (2.15) and (2.17), the solution to (2.14) can be obtained in the following form:

$$
\left.\begin{array}{c}
v_{2}=u_{0}\left(y_{2}\right) \frac{\partial A}{\partial x_{2}}\left(x_{2}, z_{2}, t_{1}\right), \\
u_{2}=-\frac{\partial u_{0}}{\partial y_{2}}\left(y_{2}\right) A\left(x_{2}, z_{2}, t_{2}\right)+C_{1}\left(y_{2}, z_{2}, t_{1}\right), \\
T_{2}=-\frac{\partial T_{0}}{\partial y_{2}}\left(y_{2}\right) A\left(x_{2}, z_{2}, t_{2}\right)+C_{2}\left(y_{2}, z_{2}, t_{1}\right),
\end{array}\right\}
$$

with functions $A, C_{1}$ and $C_{2}$ to be determined from the boundary conditions. Assuming an unperturbed solution in the incoming flow we obtain $C_{1}=C_{2}=0$. Furthermore, $\partial A / \partial x_{2} \rightarrow 0$ and $A \rightarrow 0$ as $x \rightarrow-\infty$. Then, from the boundary conditions for the temperature and velocity at the free surface, it follows that $A=F_{2}$. An expression for the perturbation of $w$ can be obtained directly from (2.14) using the pressuredisplacement law (2.19). Finally we obtain the solution in the upper inviscid layer written in terms of the free-surface displacement

$$
\left.\begin{array}{c}
v_{2}=3\left(y_{2}-\frac{y_{2}^{2}}{2}\right) \frac{\partial F_{2}}{\partial x_{2}}, \quad u_{2}=-3\left(1-y_{2}\right) F_{2}, \\
w_{2}=-\frac{K}{3\left(y_{2}-\frac{y_{2}^{2}}{2}\right)}, \quad T_{2}=-F_{2},
\end{array}\right\}
$$

where

$$
K\left(x_{2}, z_{2}, t_{1}\right)=\int_{-\infty}^{x} \frac{\partial F_{2}\left(\xi, z_{2}, t_{1}\right)}{\partial z_{2}} \cos \phi+\frac{\sin \phi}{3 W e}\left(\frac{\partial^{3} F_{2}\left(\xi, z_{2}, t_{1}\right)}{\partial \xi^{2} \partial z_{2}}+\frac{\partial^{3} F_{2}\left(\xi, z_{2}, t_{1}\right)}{\partial z_{2}^{3}}\right) \mathrm{d} \xi
$$

The system (2.21) expressed in the viscous sublayer variables yields the following boundary conditions in the viscous sublayer as $y_{1} \rightarrow \infty$ :

$$
\left.\begin{array}{cc}
v_{1}=3 y_{1} \frac{\partial F_{2}}{\partial x_{2}}, & u_{1}=3 y_{1}-3 F_{2}, \\
w_{1}=-\frac{K}{3 y_{1}}, & T_{1}=y_{1}-F_{2} .
\end{array}\right\}
$$

The boundary conditions on the ice surface $\left(y_{1}=H_{1}\right)$ in the viscous sublayer scaling become

$$
\left.\begin{array}{c}
u_{1}=0, \quad v_{1}=0, \quad w_{1}=0, \quad T_{1}=0, \\
\frac{\partial T_{1}}{\partial y_{1}}=-\lambda_{i l} T_{w} \frac{1}{H_{1}+H_{0}}-a \frac{\partial H_{1}}{\partial t_{1}},
\end{array}\right\}
$$

where $a=\operatorname{Pr} \Theta_{i l} \sim 1$ for a water/ice interface. Equations (2.12) complemented with the boundary conditions given by (2.23) and (2.24) determine the solution in the viscous layer together with the displacements of the water and ice surfaces.

Since we have applied an asymptotic expansion in Froude number to the system of boundary layer equations, which itself represents an asymptotic form of the NavierStokes set of equations at large Reynolds numbers, the validity domain of this dual expansion needs to be examined. The underlying asymptotic procedure requires, first of all that $\operatorname{Re} \gg 1$ and $F r \gg 1$. Also, for a gravity-driven flow, $F r=\sqrt{\operatorname{Re}} \sin \phi / 3$. In the boundary layer equations for the vertical velocity, the $u(\partial v / \partial x) / \sqrt{R e}$ term 
was neglected as it was small compared to $(\partial P / \partial y) \sqrt{R e}$. When we introduce the double-deck scaling, the multiplier of the $u_{2}\left(\partial v_{2} / \partial x_{2}\right)$ in the $y$ momentum equation in the inviscid layer is much greater than 1 and the multiplier of $\partial P_{2} / \partial y_{2}$ is much less then 1 . However the convection term should still be much smaller than the pressure gradient term. This requirement leads to the following restriction $F^{4} / \sqrt{R e} \ll \sqrt{R e} F r^{-1}$. Therefore, in order for the double-deck expansion to be valid, it is necessary that the following conditions are satisfied (expressed in terms of $R e$ and $\phi$ )

$$
R e \gg 1, \quad \sin \phi \gg R e^{-1 / 2}, \quad \sin ^{5} \phi \ll R e^{-3 / 2} .
$$

The first inequality has to be satisfied in order for the boundary-layer approximation to be valid. The second inequality represents the condition $F r \gg 1$ required in the double-deck expansion. The third inequality has to be satisfied in order for the doubledeck expansion to be applicable within the scope of the boundary-layer equations. In order to satisfy the last two inequalities, the angle of incline should be bounded by $R e^{-1 / 2} \ll \sin \phi \ll R e^{-3 / 10}$.

\section{Linear stability analysis}

Let us consider the behaviour of the viscous-layer solution for a small perturbation of the ice surface $\left(\varepsilon \sim H_{1} \sim F_{2} \ll H_{0}\right)$. Then in the viscous layer, the leading-order expansion of the perturbed solution is given by

$$
\left.\begin{array}{c}
H_{1}=\varepsilon H_{11}, \quad v_{1}=\varepsilon v_{11}, \quad w_{1}=\varepsilon w_{11}, \\
P_{1}=P_{0}+\varepsilon P_{11}=\left(1-y_{1}\right) \cos \phi+\varepsilon P_{11}, \\
u_{1}=u_{0}+\varepsilon u_{11}=3 y_{1}+\varepsilon u_{11}, \quad T_{1}=T_{0}+\varepsilon T_{11}=y_{1}+\varepsilon T_{11} ;
\end{array}\right\}
$$

also $F_{2}=\varepsilon F_{11}$.

For the perturbations we obtain the following system of equations:

$$
\left.\begin{array}{c}
\frac{\partial u_{11}}{\partial x_{1}}+\frac{\partial v_{11}}{\partial y_{1}}+\frac{\partial w_{11}}{\partial z_{1}}=0, \\
3 y_{1} \frac{\partial u_{11}}{\partial x_{1}}+3 v_{11}=-\frac{\partial P_{11}}{\partial x_{1}}+\frac{\partial^{2} u_{11}}{\partial y_{1}^{2}}, \\
3 y_{1} \frac{\partial w_{11}}{\partial x_{1}}=-\frac{\partial P_{11}}{\partial z_{1}}+\frac{\partial^{2} w_{11}}{\partial y_{1}^{2}}, \\
3 y \frac{\partial T_{11}}{\partial x_{1}}+v_{11}=\frac{1}{P r} \frac{\partial^{2} T_{11}}{\partial y_{1}^{2}} .
\end{array}\right\}
$$

Boundary conditions for the perturbations on the outer edge of the viscous sublayer and for the ice surface are obtained from the expansion of (2.23), and (2.24) which yields

and

$$
\left.\begin{array}{c}
v_{11}=3 y_{1} \frac{\partial F_{11}}{\partial x_{1}}, \quad u_{11}=-3 F_{11}, \\
T_{11}=-F_{11}, \quad w_{11}=-\frac{K}{3 y_{1}},
\end{array}\right\} y_{1} \rightarrow \infty,
$$

respectively.

$$
\left.\begin{array}{c}
v_{11}=0, \quad u_{11}=-3 H_{11}, \quad w_{11}=0, \quad T_{11}=-H_{11}, \\
\frac{\partial T_{11}}{\partial y_{1}}=-a \frac{\partial H_{11}}{\partial t_{1}}-\frac{H_{11}}{H_{0}},
\end{array}\right\} y_{1}=0
$$


Let us take Fourier transform in $x_{1}$ and $z_{1}$ and use a wide hat to denote transforms of the functions, for example

$$
u_{11}\left(x_{1}, y_{1}, z_{1}, t_{1}\right)=\iint \widehat{u_{11}}\left(\alpha, \beta, y_{1}, t_{1}\right) \mathrm{e}^{\mathrm{i} \alpha x_{1}+\mathrm{i} \beta z_{1}} \mathrm{~d} \alpha \mathrm{d} \beta,
$$

where $\alpha$ and $\beta$ are wavenumbers in the corresponding directions. Then we obtain the following system for the transforms:

$$
\begin{gathered}
\mathrm{i} \alpha \widehat{u_{11}}+\frac{\partial \widehat{v_{11}}}{\partial y_{1}}+\mathrm{i} \beta \widehat{w_{11}}=0 \\
3 \mathrm{i} \alpha y_{1} \widehat{u_{11}}+3 \widehat{v_{11}}=-\mathrm{i} \alpha \widehat{P_{11}}+\frac{\partial^{2} \widehat{u_{11}}}{\partial y_{1}^{2}} \\
3 \mathrm{i} \alpha y_{1} \widehat{w_{11}}=-\mathrm{i} \beta \widehat{P_{11}}+\frac{\partial^{2} \widehat{w_{11}}}{\partial y_{1}^{2}}, \\
3 \mathrm{i} \alpha y_{1} \widehat{T_{11}}+\widehat{v_{11}}=\frac{1}{\operatorname{Pr}} \frac{\partial^{2} \widehat{T_{11}}}{\partial y_{1}^{2}} .
\end{gathered}
$$

The pressure-displacement law can be written in terms of the Fourier transform of the pressure function $P_{11}$ as

$$
\widehat{P_{11}}=\Phi \widehat{F_{11}}
$$

where $\Phi$ is a function of the wavenumbers $\alpha$ and $\beta$,

$$
\Phi=\cos \phi+\frac{\alpha^{2}+\beta^{2}}{W e} \frac{\sin \phi}{3} .
$$

At the leading order, the boundary conditions on the ice surface become

$$
\left.\begin{array}{c}
\widehat{u_{11}}=-3 \widehat{H_{11}}, \quad \widehat{v_{11}}=0, \quad \widehat{w_{11}}=0, \quad \widehat{T_{11}}=-\widehat{H_{11}}, \\
\frac{\partial \widehat{T_{11}}}{\partial y_{1}}=-a \frac{\partial \widehat{H_{11}}}{\partial t_{1}}-\frac{\widehat{H_{11}}}{H_{0}} .
\end{array}\right\} y_{1}=0 .
$$

Note that in numerical computations we apply boundary conditions at a cutoff point $y_{1}=y_{e}$. In this case, a better precision can be obtained if we derive one more term in the expansion for $\widehat{v_{11}}$ and use it as a correction to the boundary condition at large values of $y_{1}$. In order to do this, we write the condition for $\widehat{v_{11}}$ as $y_{1} \rightarrow \infty$ in the form $\widehat{v_{11}}=3 \mathrm{i} \alpha y_{1} \widehat{F_{11}}+X$, where $X$ is some unknown function. Then the limit of equation (3.6a) as $y_{1} \rightarrow \infty$ leads to

$$
-9 \mathrm{i} \alpha y_{1} \widehat{F_{11}}+9 \mathrm{i} \alpha y_{1} \widehat{F_{11}}+3 X=-\mathrm{i} \alpha \widehat{P_{11}}+0 .
$$

Hence the second term in the expansion for $\widehat{v_{11}}$ does not depend on $y_{1}$ and the boundary condition for $\widehat{v_{11}}$ as $y_{1} \rightarrow \infty$ becomes

$$
\widehat{v_{11}}=3 \mathrm{i} \alpha y_{1} \widehat{F_{11}}-\frac{\mathrm{i} \alpha}{3} \widehat{F_{11}} \Phi .
$$

Also, from the solution for $w_{2}$ in the inviscid layer given by (2.21) it follows that, in terms of Fourier transforms, the boundary condition for $\widehat{w_{11}}$ as $y_{1} \rightarrow \infty$ is given by

$$
\widehat{w_{11}}=-\frac{\beta}{3 \alpha} \widehat{F_{11}} \Phi \frac{1}{y_{1}} .
$$

Finally, with the correction for $\widehat{v_{11}}$ taken into account, the complete set of boundary conditions for Fourier transforms at the outer edge of the viscous layer can be 
written as

$$
\left.\begin{array}{c}
\widehat{u_{11}}=-3 \widehat{F_{11}}, \quad \widehat{v_{11}}=3 \mathrm{i} \alpha y_{1} \widehat{F_{11}}-\frac{\mathrm{i} \alpha}{3} \widehat{F_{11}} \Phi, \\
\widehat{w_{11}}=-\frac{\beta}{3 \alpha} \widehat{F_{11}} \Phi \frac{1}{y_{1}}, \quad \widehat{T_{11}}=-\widehat{F_{11}} .
\end{array}\right\} y_{1} \rightarrow \infty
$$

3.1. Analytic solution, $\operatorname{Pr}=1$

When the base-flow velocity profile is linear, the Orr-Sommerfeld equation contains solutions in terms of Airy functions (for example, see Hooper \& Boyd 1983). In our case, equations for $\widehat{u_{11}}$ and $\widehat{T_{11}}$ are similar when $\operatorname{Pr}=1$ and the solution can be obtained analytically in terms of Airy functions for the system with temperature as well.

The equation for $\widehat{w_{11}}$ in (3.2) can be rewritten in the form of the Scorer equation. By setting

$$
M(\zeta)=\widehat{w_{11}}\left(y_{1}\right), \quad \zeta=(3 \mathrm{i} \alpha)^{1 / 3} y_{1} \quad \text { and } \quad \eta(\alpha, \beta)=\frac{\mathrm{i} \beta}{(3 \mathrm{i} \alpha)^{2 / 3}} \widehat{P_{11}}=\frac{\mathrm{i} \beta}{(3 \mathrm{i} \alpha)^{2 / 3}} \widehat{F_{11}} \Phi
$$

we obtain

$$
\frac{\partial^{2} M}{\partial \zeta^{2}}-\zeta M=\eta(\alpha, \beta)
$$

The general solution of equation (3.14) can be written in the form

$$
M(\zeta)=C_{1} M_{1}(\zeta)+C_{2} M_{2}(\zeta)-\pi \eta(\alpha, \beta) M_{3}(\zeta)
$$

Here $M_{1}$ and $M_{2}$ are two linearly independent solutions of the Airy equation and $M_{3}$ is any particular solution of the Scorer equation. Taking $\operatorname{Ai}(\zeta), \operatorname{Bi}(\zeta)$ and $\operatorname{Gi}(\zeta)$ as a group of solutions for the Scorer equation (see, for example, Abramowitz \& Stegun 1972) and noticing that $\mathrm{Bi}$ is not bounded at infinity, (3.15) can be reduced to

$$
M(\zeta)=C_{1} \operatorname{Ai}(\zeta)-\pi \eta(\alpha, \beta) \operatorname{Gi}(\zeta)
$$

From the boundary condition for $\widehat{w_{11}}$ on the ice surface, it follows that $M(0)=0$. Therefore

$$
C_{1}=\pi \eta(\alpha, \beta) \frac{\mathrm{Gi}(0)}{\operatorname{Ai}(0)}=\pi \eta(\alpha, \beta) \frac{1}{\sqrt{3}} .
$$

Then for $\widehat{w_{11}}$, we obtain the following solution:

$$
\widehat{w_{11}}=\left(\frac{\operatorname{Ai}(\zeta)}{\sqrt{3}}-\mathrm{Gi}(\zeta)\right) \pi \frac{\mathrm{i} \beta}{(3 \mathrm{i} \alpha)^{2 / 3}} \widehat{F_{11}} \Phi .
$$

If we multiply the partial derivative with respect to $y_{1}$ of equation (3.6a) by i $\alpha$, add it to the partial derivative with respect to $y_{1}$ of equation (3.6b) multiplied by $\mathrm{i} \beta$ and use the expression for $\partial \widehat{v_{11}} / \partial y_{1}$ from the continuity equation, we obtain the following equation for $\widehat{v_{11}}$ :

$$
\frac{\partial^{4} \widehat{v_{11}}}{\partial y_{1}^{4}}=3 i \alpha y_{1} \frac{\partial^{2} \widehat{v_{11}}}{\partial y_{1}^{2}},
$$

which constitutes an Airy equation for the second derivative $\partial^{2} \widehat{v_{11}} / \partial y_{1}^{2}$. The solution of (3.18) yields

$$
\frac{\partial^{2} \widehat{v_{11}}}{\partial y_{1}^{2}}=W(\alpha, \beta) \operatorname{Ai}(\zeta)
$$

where the function $W$ is to be determined from the boundary conditions. 
Using (3.17), (3.19) and the continuity equation differentiated by $y_{1}$, we obtain the following expression for the first derivative of the $x_{1}$ component of velocity:

$$
\begin{aligned}
\frac{\partial \widehat{u_{11}}}{\partial y_{1}} & =-\frac{1}{\mathrm{i} \alpha}\left(\frac{\partial^{2} \widehat{v_{11}}}{\partial y_{1}^{2}}+\mathrm{i} \beta \frac{\partial \widehat{w_{11}}}{\partial y_{1}}\right) \\
& =-\frac{1}{\mathrm{i} \alpha}\left(W \operatorname{Ai}(\zeta)+\mathrm{i} \beta\left(\frac{1}{\sqrt{3}} \frac{\mathrm{dAi}(\zeta)}{\mathrm{d} \zeta}-\frac{\mathrm{dGi}(\zeta)}{\mathrm{d} \zeta}\right) \frac{\mathrm{i} \beta \pi \widehat{F_{11}} \Phi}{(3 \mathrm{i} \alpha)^{1 / 3}}\right) .
\end{aligned}
$$

Then we note that if $P r=1$, equations (3.6b) and (3.6d) are similar. If we multiply the equation for temperature by 3 and subtract the equation for the $x_{1}$ component of velocity from it, we obtain

$$
3 i \alpha y_{1}\left(3 \widehat{T_{11}}-\widehat{u_{11}}\right)-\mathrm{i} \alpha \widehat{P_{11}}=\frac{\partial^{2}\left(3 \widehat{T_{11}}-\widehat{u_{11}}\right)}{\partial y_{1}^{2}} .
$$

Clearly, (3.21) represents a Scorer equation for $\left(3 \widehat{T_{11}}-\widehat{u_{11}}\right)$ in terms of the variable $\zeta$ and its solution is given by

$$
3 T-u=\pi\left(\frac{\operatorname{Ai}(\zeta)}{\sqrt{3}}-\operatorname{Gi}(\zeta)\right) \frac{(\mathrm{i} \alpha)^{1 / 3}}{3^{2 / 3}} F \Phi .
$$

Equations (3.17), (3.19), (3.20) and (3.22) allow us to obtain solution for $\widehat{u_{11}}, \widehat{v_{11}}$, $\widehat{w_{11}}$ and $\widehat{T_{11}}$ in terms of the unknown function $W$, and Fourier transforms of the ice-surface displacement $\widehat{H_{11}}$ and free-surface displacement $\widehat{F_{11}}$. Now let us use all the boundary conditions apart from the Stefan condition to derive equations linking $\widehat{H_{11}}$, $\widehat{F_{11}}$ and $W$, which we can use to reduce the Stefan condition to a differential equation containing the ice-surface displacement alone.

From the equations for $\widehat{u_{11}}$ and $\widehat{w_{11}}$ in the system (3.6d) taken at $y_{1}=0$ we obtain the following expressions for the second derivatives of $\widehat{u_{11}}$ and $\widehat{w_{11}}$ :

$$
\begin{aligned}
& \frac{\partial^{2} \widehat{u_{11}}}{\partial y_{1}^{2}}(0)=\mathrm{i} \alpha \widehat{F_{11}} \Phi, \\
& \frac{\partial^{2} \widehat{w_{11}}}{\partial y_{1}^{2}}(0)=\mathrm{i} \beta \widehat{F_{11}} \Phi .
\end{aligned}
$$

Substituting (3.23) and (3.24) into the expression for the third derivative of $\widehat{v_{11}}$ derived from (3.19) we obtain

$$
\frac{\partial^{3} \widehat{v_{11}}}{\partial y_{1}^{3}}(0)=(3 \mathrm{i} \alpha)^{1 / 3} W \frac{\mathrm{dAi}(\zeta)}{\mathrm{d} \zeta}(0)=-\mathrm{i} \alpha \frac{\partial^{2} \widehat{u_{11}}}{\partial y_{1}^{2}}-\mathrm{i} \beta \frac{\partial^{2} \widehat{w_{11}}}{\partial y_{1}^{2}}=\widehat{F_{11}} \Phi\left(\alpha^{2}+\beta^{2}\right),
$$

which gives us the first relation between $\widehat{F_{11}}$ and $W$ :

$$
W=X_{0} \widehat{F_{11}} \text {, }
$$

where

$$
X_{0}=\left(\alpha^{2}+\beta^{2}\right) \frac{\Phi}{\frac{\mathrm{dAi}(\zeta)}{\mathrm{d} \zeta}(0)(3 \mathrm{i} \alpha)^{1 / 3}} .
$$


Next, let us consider the integral from 0 to $\infty$ in $y_{1}$ of $\partial^{2} \widehat{v_{11}} / \partial y_{1}^{2}$ given by (3.19) with the appropriate boundary conditions (see (3.9) and (3.13))

$$
\int_{0}^{\infty} \frac{\partial^{2} \widehat{v_{11}}}{\partial y_{1}^{2}} \mathrm{~d} y_{1}=-\mathrm{i} \alpha \widehat{u_{11}}(\infty)+i \alpha \widehat{u_{11}}(0)=3 \mathrm{i} \alpha\left(\widehat{F_{11}}-\widehat{H_{11}}\right) .
$$

At the same time from (3.19) we have

$$
\int_{0}^{\infty} \frac{\partial^{2} \widehat{v_{11}}}{\partial y_{1}^{2}} \mathrm{~d} y_{1}=\frac{1}{3}(3 \mathrm{i} \alpha)^{-1 / 3} W
$$

Equating (3.28) and (3.29) and using (3.26) to eliminate $W$, we obtain the second equation linking $\widehat{F_{11}}$ and $\widehat{H_{11}}$ :

$$
\widehat{F_{11}}=\mathrm{X}_{1} \widehat{H_{11}}
$$

where

$$
X_{1}=\left(1-\frac{\Phi\left(\alpha^{2}+\beta^{2}\right)}{3 \frac{\mathrm{dAi}(\zeta)}{\mathrm{d} \zeta}(0)(3 \mathrm{i} \alpha)^{5 / 3}}\right)^{-1}
$$

Now we can differentiate (3.22) by $y_{1}$ and use the solution for $\partial \widehat{u_{11}} / \partial y_{1}$ given by (3.20) to eliminate $\partial \widehat{T_{11}} / \partial y_{1}$ from the Stefan condition. Then, if we eliminate $\widehat{F_{11}}$ and $W$ using (3.26) and (3.30) respectively, we finally obtain a differential equation defining the evolution of the Fourier transform of the ice surface in the following form:

$$
\frac{\partial \widehat{H}_{11}}{\partial t_{1}}=G\left(\phi, \alpha, \beta, H_{0}, a\right) \widehat{H_{11}},
$$

where the complex growth rate $G$ is given by

$$
G=\frac{X_{1}}{3 a \mathrm{i} \alpha}\left(\pi \frac{2(3 \mathrm{i} \alpha)^{5 / 3}}{3^{5 / 2}} \Phi \frac{\mathrm{dAi}(\zeta)}{\mathrm{d} \zeta}(0)-\frac{3 \mathrm{i} \alpha}{H_{0} X_{1}}+X_{0} \mathrm{Ai}(0)-\frac{2 \frac{\mathrm{dAi}(\zeta)}{\mathrm{d} \zeta}(0) \pi \beta^{2}}{\sqrt{3}(3 \mathrm{i} \alpha)^{1 / 3}} \Phi\right)
$$

and $\Phi, X_{0}$ and $X_{1}$ are defined by (3.8), (3.27) and (3.31) respectively.

The real part of the complex growth rate is given by $w_{i}=\operatorname{Re}(G)$ and the real phase speed of the wave travelling with the wavenumber vector $(\alpha, \beta)$ is given by $c_{r}=-\operatorname{Im}(G) / \sqrt{\alpha^{2}+\beta^{2}}$. The formula for the growth rate of two-dimensional disturbances can be obtained by taking the limit of (3.33) as $\beta \rightarrow 0$. Note that from the formula for the complex growth rate it can be seen immediately that the Prandt number and the water/ice density ratio do not influence neutral curves of the linear problem.

The neutral stability domain is given by $w_{i}=\operatorname{Re}(G)=0$. For specified ice/water properties and the angle of incline $\phi$, the neutral curve represents a surface in $\left(\alpha, \beta, H_{0}\right)$ space which is given by the following expression:

$$
H_{0}(\alpha, \beta, W e, \phi)=2 \frac{1-\sqrt{3} A_{2}+A_{2}^{2}}{-1+\sqrt{3} A_{2}} \frac{1}{-A_{1}+A_{3}+A_{4}},
$$



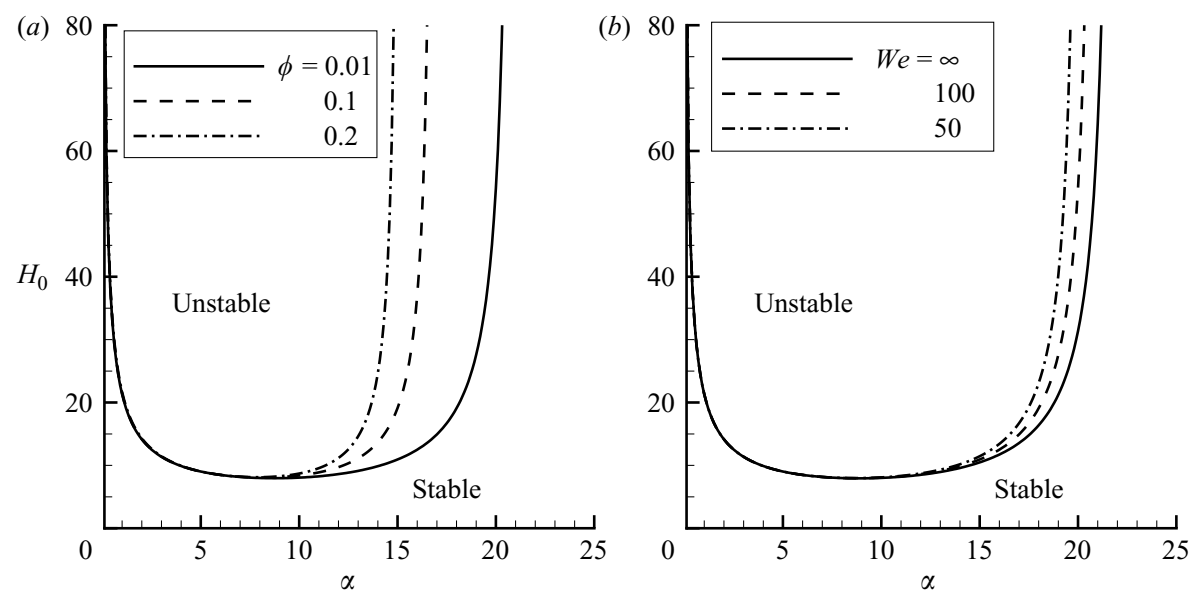

FIgURE 2. Two-dimensional neutral curves for $\operatorname{Pr}=1$. (a) Dependence on $\phi(\mathrm{We}=100)$. (b) Dependence on Weber number $(\phi=0.01)$.

where coefficient functions $A_{1-4}$ are defined as follows:

$$
\begin{gathered}
A_{1}(\alpha, \beta, W e, \phi)=2 \pi 3^{-5 / 6} \alpha^{2 / 3} \frac{\mathrm{dAi}(\zeta)}{\mathrm{d} \zeta}(0) \\
A_{2}(\alpha, \beta, W e, \phi)=-(3 \alpha)^{-5 / 3} \frac{\alpha^{2}+\beta^{2}}{\frac{\mathrm{dAi}(\zeta)}{\mathrm{d} \zeta}(0)}\left(\cos \phi+\frac{\alpha^{2}+\beta^{2}}{W e} \frac{\sin \phi}{3}\right) \\
A_{3}(\alpha, \beta, W e, \phi)=\frac{\operatorname{Ai}(0)}{\frac{\mathrm{dAi}(\zeta)}{\mathrm{d} \zeta}(0)} \frac{\alpha^{2}+\beta^{2}}{3^{1 / 3} \alpha^{4 / 3}}\left(\cos \phi+\frac{\alpha^{2}+\beta^{2}}{W e} \frac{\sin \phi}{3}\right) \\
A_{4}(\alpha, \beta, W e, \phi)=-2 \frac{\mathrm{dAi}(\zeta)}{\mathrm{d} \zeta}(0) \frac{\pi \beta^{2}}{3^{5 / 6} \alpha^{4 / 3}}\left(\cos \phi+\frac{\alpha^{2}+\beta^{2}}{W e} \frac{\sin \phi}{3}\right)
\end{gathered}
$$

An expression for the neutral curve in two-dimensional flow can be obtained by taking the limit of (3.34) as $\beta \rightarrow 0$. In the two-dimensional case, it is convenient to analyse neutral curves in the $\left(H_{0}, \alpha\right)$ parameter plane for fixed values of the angle of incline and the Weber number. Figure 2 shows two-dimensional neutral curves in this plane for varying angle of incline (figure $2 a$ ) and inverse of the Weber number (figure $2 b$ ) respectively. Note that under the double-deck assumptions we have $W e \gg 1$ in the water flow. A decrease in the Weber number or an increase in the angle of incline lead to damping of short-wave disturbances.

An important property of icing instability immediately visible from the twodimensional neutral curves is that there exists a limiting value of the initial ice height below which the mode is stable to two-dimensional disturbances. Similar qualitative behaviour has been observed in channel icing experiments by Gilpin (1979, 1981).

From the form of the expression for the complex growth rate (3.33) it follows that the value of $H_{0}$ has no influence on the phase speed. Figure 3 shows the growth rate and the real part of the complex phase speed as functions of the wavenumber $\alpha$ for different values of the initial ice thickness $H_{0}$ in the two-dimensional case.

Note that the sign of $c_{r}$ is negative. This indicates that the ice-surface perturbation may propagate upstream, at least when in the form of harmonic waves. However, 

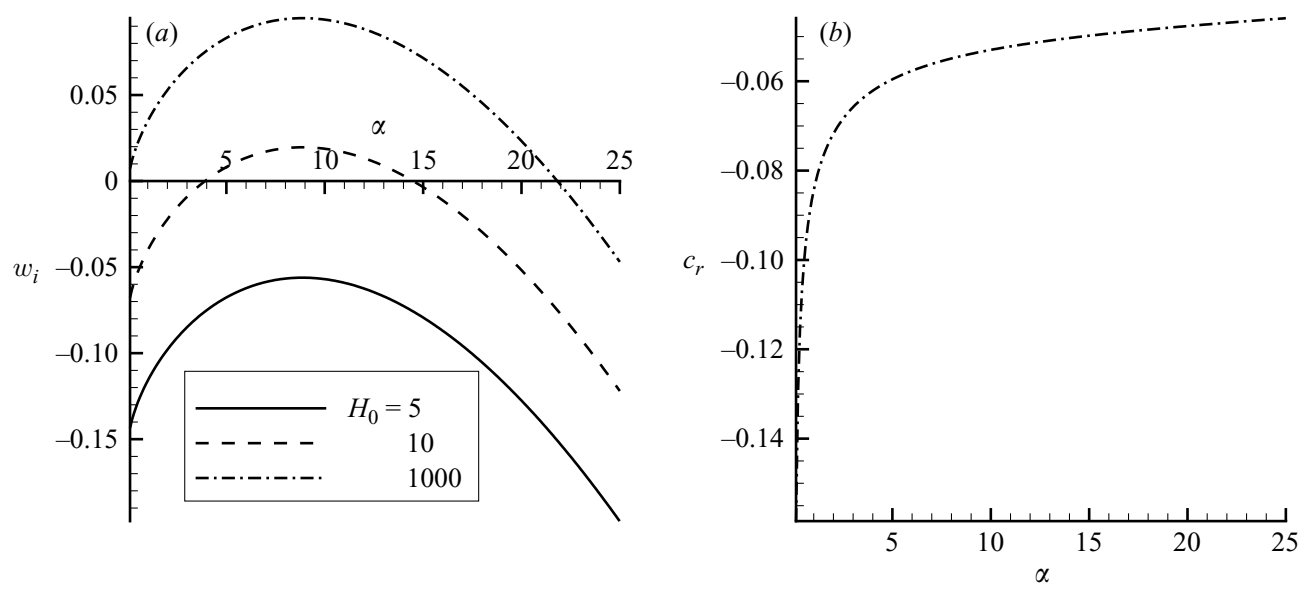

FIGURE 3. Two-dimensional growth rate and phase for $\phi=0.001$ and $W e=1000:(a) w_{i},(b) c_{r}$.
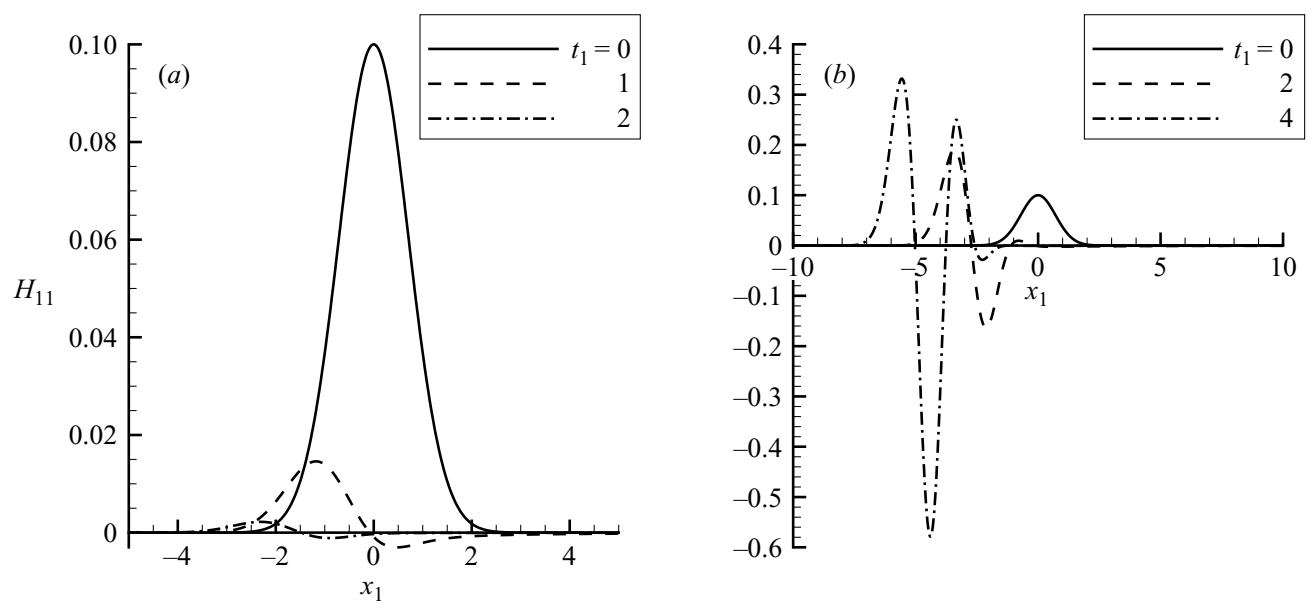

FiguRE 4. $H_{11}\left(t_{1}\right)$, two-dimensional case for $\phi=0.001$ and $W e=1000$. (a) Stable case, $H_{0}=5$. (b) Unstable case, $H_{0}=30$.

in order to establish whether the same is also true for wave-packet perturbations, one either has to perform the spatio-temporal analysis described, for example, in Briggs (1964) or, alternatively, consider the evolution of a spatially localized initial disturbance. We have employed the latter method and considered the evolution of a small Gaussian disturbance to the ice shape, $H_{\text {init }}=\varepsilon \mathrm{e}^{-x^{2}}$. The real function for $H_{11}$ was obtained by inverting the Fourier transform and computing the resulting integral numerically. Computations performed for the chosen form of the initial disturbance demonstrate that a disturbance introduced into the flow under unstable conditions grows and propagates upstream, whereas disturbance introduced under stable conditions decays, but still propagates upstream. The behaviour of growing and decaying solutions is illustrated in figure 4 for $\varepsilon=0.1$.

In the three-dimensional case, the neutral curve is a surface in the $\left(\alpha, \beta, H_{0}\right)$ space. It is convenient then to examine the contours of this surface for fixed values of $H_{0}$. Figure 5 shows the variation of the three-dimensional neutral stability curve with the 

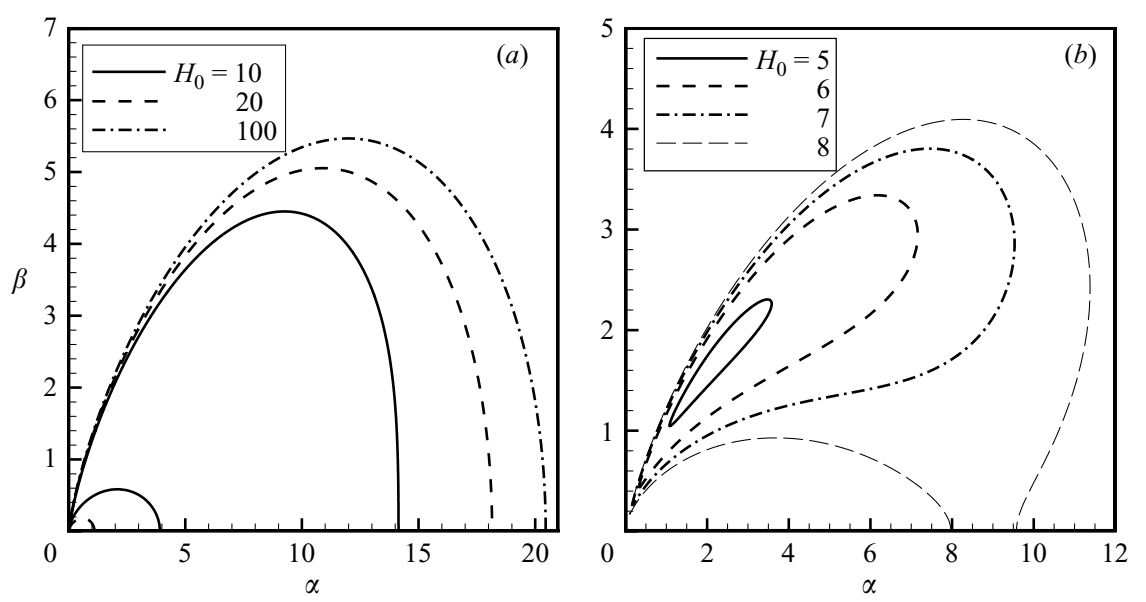

FiguRE 5. Three-dimensional neutral curve dependence on $H_{0}(\phi=0.01, W e=100)$; $(a)$ large $H_{0}$, (b) small $H_{0}$.
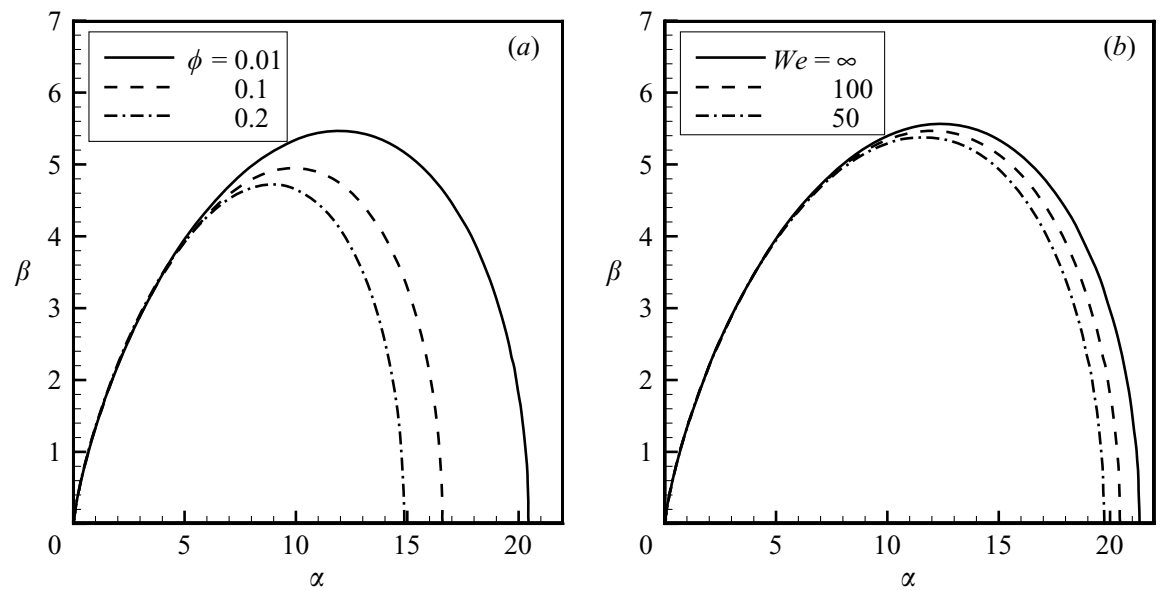

FIGURE 6. Three-dimensional neutral curve dependence on $\phi$ and We: $(a)$ dependence on $\phi\left(H_{0}=100, W e=100\right),(b)$ dependence on $W e\left(H_{0}=100, \phi=0.01\right)$.

change of $H_{0}$ for large (figure 5) and small (figure 5) values of $H_{0}$, respectively. As in the two-dimensional case, the domain of unstable wavenumbers increases as we increase the initial ice height; however, there is a limiting curve which is approached rapidly (there is no visible difference in the curves for $H_{0}=100$ and $H_{0}=1000$ ). Furthermore, from figure $5(b)$ it can be seen that there is a range of $H_{0}$ values where the ice surface becomes unstable to three-dimensional disturbances before becoming unstable to two-dimensional disturbances, i.e. if the ice layer grows from zero initial thickness, the initial evolution of the ice surface roughness occurs at an angle to the flow direction. This behaviour was observed experimentally by Streitz \& Ettema (2002).

The effect of the Weber number and the angle of incline is essentially the same as in the two-dimensional case, with the increase of the angle of incline or decrease of the Weber number leading to increased impact of surface tension, thus suppressing short-wave disturbances (see figure 6). 


\subsection{Numerical solution for $\operatorname{Pr} \neq 1$}

In the general case $\operatorname{Pr} \neq 1$ and equations for temperature and horizontal velocity cannot be combined, but the solutions for Fourier transforms $\widehat{u_{11}}, \widehat{v_{11}}$ and $\widehat{w_{11}}$ (which can be obtained from equations (3.20), (3.19) and (3.17) respectively) are still valid. In this case, it is possible to use the solution for the velocity field in terms of the Airy functions and solve the equation for temperature numerically. This method leads to a Scorer-type equation for temperature with a non-constant source term, which contains a double integral of the Airy function Ai. On the other hand, if we build a numerical solver for the Airy-type equations, then it is easy to solve the whole problem numerically without reducing it to a single temperature equation, and thus avoid the overhead introduced by double numerical integration of the Airy function. This approach is more efficient than solving the temperature equation directly.

The system of linear stability equations for the double-deck flow can be converted into a system of second-order differential equations by introducing the following set of functions:

$$
V=\frac{\widehat{v_{11}}}{\widehat{F_{11}}}-3 i \alpha y_{1}, \quad V_{I I}=\frac{1}{\widehat{F_{11}}} \frac{\partial^{2} \widehat{v_{11}}}{\partial y_{1}^{2}}, \quad \theta=1+\frac{\widehat{T_{11}}}{\widehat{F_{11}}}
$$

Then we obtain the following system of equations for $V, V_{I I}$ and $\theta$ :

$$
\frac{\partial^{2} V_{I I}}{\partial y_{1}^{2}}=3 \mathrm{i} \alpha y_{1} V_{I I}, \quad \frac{\partial^{2} V}{\partial y_{1}^{2}}=V_{I I}, \quad \frac{\partial^{2} \theta}{\partial y_{1}^{2}}=3 \mathrm{i} \alpha y_{1} \theta+V,
$$

with boundary conditions given by

$$
\left.\begin{array}{c}
\frac{\partial V_{I I}}{\partial y_{1}}(0)=\left(\alpha^{2}+\beta^{2}\right)\left(\cos \phi+\frac{\alpha^{2}+\beta^{2}}{W e} \frac{\sin \phi}{3}\right), \\
V(0)=0, \quad \theta(0)=1-\frac{1}{X_{1}}, \quad V_{I I}(\infty)=0, \\
V(\infty)=-\frac{\mathrm{i} \alpha}{3}\left(\cos \phi+\frac{\alpha^{2}+\beta^{2}}{W e} \frac{\sin \phi}{3}\right), \\
\theta(\infty)=0 .
\end{array}\right\}
$$

Once the numerical solution of (3.40)-(3.41) is known, we can compute the value of $\partial \theta / \partial y_{1}(0)$ which is needed to solve the evolution equation for the ice-surface displacement $\widehat{H_{11}}$. The latter can be written in terms of the function $\theta$ as

$$
\frac{\partial \widehat{H_{11}}}{\partial t_{1}}=-\widehat{H_{11}}\left(X_{1} \frac{\partial \theta}{\partial y_{1}}(0)+\frac{1}{H_{0}}\right) .
$$

The real ice-surface perturbation function $H_{11}\left(x_{1}, z_{1}, t_{1}\right)$ can then be obtained by taking the inverse Fourier transform of $\widehat{H_{11}}\left(\alpha, \beta, t_{1}\right)$. Also, since the coefficient of $\widehat{H_{11}}$ on the right-hand side of (3.42) does not depend on $t_{1}$, the system (3.40)-(3.41) need be solved only once on a chosen grid in $(\alpha, \beta)$ space, then the resulting matrix of coefficients can be used to determine $\widehat{H_{11}}\left(x_{1}, z_{1}, t_{1}\right)$ for any $t_{1}$.

We employed a standard second-order-accurate central differences approximation of the derivatives in order to discretize the second-order system (3.40)-(3.41). The resulting 3-diagonal linear system of equations was solved using forwards-backwards substitution (see, for example, Isaacson \& Keller 1966). The original function $H_{11}\left(x_{1}, z_{1}, t_{1}\right)$ was then obtained from the Fourier image by a direct numerical integration. 

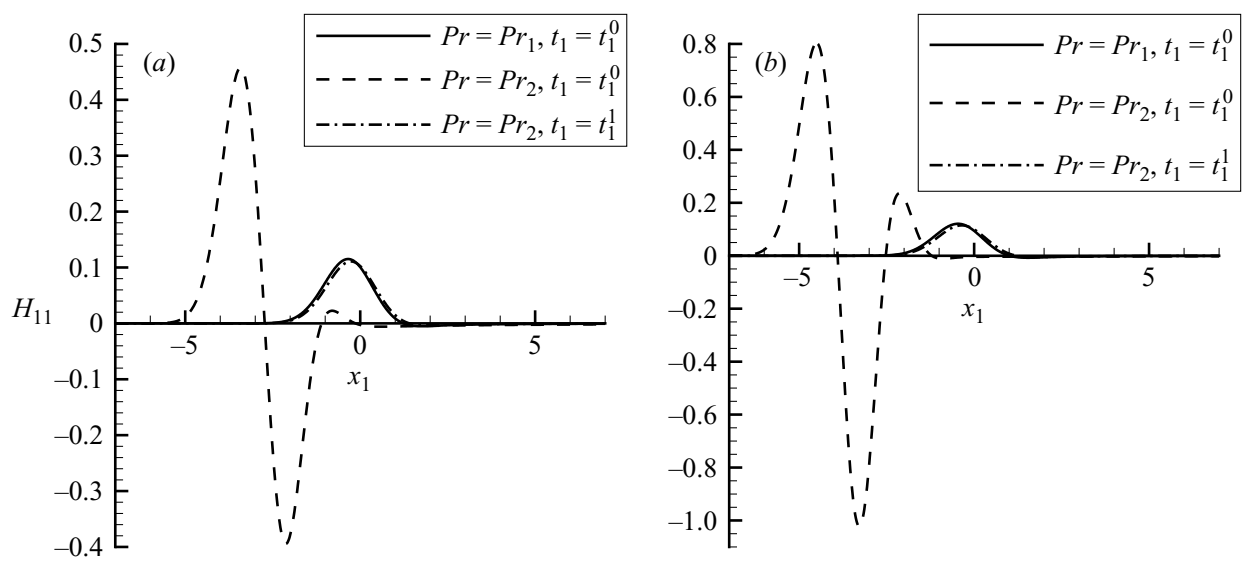

Figure 7. The dependence of the linear problem solution on the Prandtl number $\left(H_{0}=100\right.$, $\phi=0.001$ and $W e=1000)(a) t=2,(b) t=5$.

The numerically determined approximation order in $y_{1}$ was found to be 1.992 on a grid of 1000 nodes in $y_{1}$ and improved on finer grids. The upper boundary conditions have to be set at some finite cutoff distance $y_{1}=y^{e}$; the numerically determined convergence in $y^{e}$ was $\sim y^{e} / M$, where $M=$ const $<10^{-3}$ for $y^{e}>10$. Based on the grid and cutoff value convergence studies, we have chosen an equally spaced grid of 2000 points in $y_{1}$ with $y^{e}=20$, which was used to compute all results reported in this section.

With the numerical solution to hand, the obvious question to ask is how relevant is our exact solution with $\mathrm{Pr}=1$. In other words, how different is the solution for the Prandtl number for water $\left(\sim 13.47\right.$ at $\left.0^{\circ} \mathrm{C}\right)$ from the solution for $P r=1$ ? In order to analyse the influence of the Prandtl number, we note that it appears in the equations only in two places, namely in the advection-diffusion equation for the temperature (3.6d) and in the Stefan condition on the ice surface (3.9). In the latter case it appears as the multiplier of the only time derivative left in the system, and can be removed by rescaling the time. With this in mind let us consider the following three cases:

(a) $P r_{1}=13.47$, water at $0^{\circ} \mathrm{C}$, computed numerically at time $t_{1}=t_{1}^{0}$;

(b) $P r_{2}=1.0$, analytic solution at $t_{1}=t_{1}^{0}$;

(c) $P r_{2}=1.0$, analytic solution at $t_{1}=t_{1}^{1}=t_{1}^{0} P r_{2} / P r_{1}$.

A comparison between these three cases for a two-dimensional disturbance is shown in figure 7 (computations for other values of the parameters yielded similar results, therefore we use only one point in the parameter space as an illustration). It can be clearly seen that the analytic solution for $P r=1$ with rescaled time differs negligibly from the numeric solution for $P r=13.47$. This demonstrates that the major influence of the Prandtl number is in the growth rate coefficient and the analytic solution can be used when $\operatorname{Pr} \neq 1$, provided we rescale the time accordingly. This result essentially means that in the linear problem, the thermal boundary layer affects the speed of disturbance propagation but has no significant impact on the shape of disturbances. The behaviour of three-dimensional disturbances was found to be similar.

\section{Nonlinear two-dimensional flow}

We shall now address two-dimensional nonlinear flow regimes in the viscous layer. As in the linear case, we can derive an exact solution for the temperature distribution 
in the ice layer. Using this solution to eliminate the ice temperature from the boundary conditions on the ice surface, we obtain a boundary-value problem for the nonlinear viscous double-deck flow in the following form:

$$
\begin{gathered}
\frac{\partial u_{1}}{\partial x_{1}}+\frac{\partial v_{1}}{\partial y_{1}}=0, \\
u_{1} \frac{\partial u_{1}}{\partial x_{1}}+v_{1} \frac{\partial u_{1}}{\partial y_{1}}=-\frac{\partial P_{1}}{\partial x_{1}}+\frac{\partial^{2} u_{1}}{\partial y_{1}^{2}}, \\
u_{1} \frac{\partial T_{1}}{\partial x_{1}}+v_{1} \frac{\partial T_{1}}{\partial y_{1}}=\frac{1}{P r} \frac{\partial^{2} T_{1}}{\partial y_{1}^{2}}, \\
P_{1}=F_{2} \cos \phi-\frac{\partial^{2} F_{2}}{\partial x_{1}^{2}} \frac{\sin \phi}{3 W e}, \\
u_{1}\left(H_{1}\right)=0, \quad u_{1}(\infty)=3 y_{1}-3 F_{2}, \\
v_{1}\left(H_{1}\right)=0, \quad v_{1}(\infty)=3 y_{1} \frac{\partial F_{2}}{\partial x_{1}}, \\
T_{1}\left(H_{1}\right)=0, \quad T_{1}(\infty)=y_{1}-F_{2}, \\
\frac{\partial T_{1}}{\partial y_{1}}\left(H_{1}\right)=-\lambda_{i l} \frac{T_{w}}{H_{1}+H_{0}}-a \frac{\partial H_{1}}{\partial t_{1}} .
\end{gathered}
$$

The formulation above is similar to those arising in the triple-deck theory of boundary-layer flows at large Reynolds numbers. Therefore it seems reasonable to employ numerical approaches that have been successfully applied in the triple-deck theory for steady and unsteady problems of boundary-layer separation. We derive a global-marching-type scheme for this problem, similar to the schemes used by Cassel, Ruban \& Walker (1995), but based on the conservation form of the equations. In order to do this we introduce derivatives in pseudo-time $\tau$ to construct an iterative process and rewrite our equations in the conservative form (see for example Chung 2002) in Cartesian coordinates as

$$
\frac{\partial \boldsymbol{Q}_{c}}{\partial \tau}+\frac{\partial \boldsymbol{E}_{c}}{\partial x_{1}}+\frac{\partial \boldsymbol{G}_{c}}{\partial y_{1}}=0,
$$

where the fluxes are given by

$$
\begin{gathered}
\boldsymbol{E}_{c}=\left\|\begin{array}{c}
u_{1}^{2}+P_{1} \\
u_{1} T_{1}
\end{array}\right\|, \\
\boldsymbol{G}_{c}=\left\|\begin{array}{c}
u_{1} v_{1}-\frac{\partial u_{1}}{\partial y_{1}} \\
v_{1} T_{1}-\frac{1}{P r} \frac{\partial T_{1}}{\partial y_{1}}
\end{array}\right\|,
\end{gathered}
$$

and

$$
\boldsymbol{Q}_{c}=\left\|\begin{array}{l}
Q_{c 1} \\
Q_{c 2}
\end{array}\right\|
$$

is a function used to construct iterations which can be varied in order to obtain better convergence. Similarly to Cassel et al. (1995), we apply arctangent transform to map our computational domain onto a rectangle $(-1 . .+1,0 . .+1)$ and solve the equations 
in the transformed $(\xi, \eta)$ space defined by

$$
\xi=\frac{2}{\pi} \arctan \left(\frac{x_{1}-x_{10}}{A}\right), \quad \eta=\frac{2}{\pi} \arctan \left(\frac{y_{1}-H_{1}\left(x_{1}, t_{1}\right)}{B}\right),
$$

where $x_{10}$ gives the centre of grid compression. Constants $A$ and $B$ can be used to adjust grid concentration. The significant difference is that in our case the transform is time-dependent, i.e. we are solving the flow equations on a moving grid. However the Jacobian of the transformation $J$ given by

$$
J(\xi, \eta)=\frac{\partial\left(x_{1}, y_{1}\right)}{\partial(\xi, \eta)}=A B\left(\frac{\pi}{2}\right)^{2} \frac{1}{\cos ^{2}\left(\frac{\pi}{2} \xi\right) \cos ^{2}\left(\frac{\pi}{2} \eta\right)}
$$

does not depend on time, which simplifies computations. The governing equations retain their conservative form in the $(\xi, \eta)$-space:

$$
\frac{\partial \boldsymbol{Q}}{\partial \tau}+\frac{\partial \boldsymbol{E}}{\partial \xi}+\frac{\partial \boldsymbol{G}}{\partial \eta}=0
$$

where $\boldsymbol{Q}=J \boldsymbol{Q}_{c}$ and the fluxes are given by (taking into account the form of (4.6))

$$
\boldsymbol{E}=J(\xi, \eta) \frac{\partial \xi}{\partial x_{1}}\left\|\begin{array}{c}
u_{1}^{2}+P_{1} \\
u_{1} T_{1}
\end{array}\right\|
$$

and

$$
\boldsymbol{G}=J(\xi, \eta)\left(\frac{\partial \eta}{\partial x_{1}}\left\|\begin{array}{c}
u_{1}^{2}+P_{1} \\
u_{1} T_{1}
\end{array}\right\|+\frac{\partial \eta}{\partial y_{1}}\left\|\begin{array}{c}
u_{1} v_{1}-\frac{\partial \eta}{\partial y_{1}} \frac{\partial u}{\partial \eta} \\
T_{1} v_{1}-\frac{1}{\operatorname{Pr}} \frac{\partial \eta}{\partial y_{1}} \frac{\partial T}{\partial \eta}
\end{array}\right\|\right)
$$

The continuity equation in the new variables becomes

$$
\frac{\partial \xi}{\partial x_{1}} \frac{\partial u_{1}}{\partial \xi}+\frac{\partial \eta}{\partial x_{1}} \frac{\partial u_{1}}{\partial \eta}+\frac{\partial \eta}{\partial y_{1}} \frac{\partial v_{1}}{\partial \eta}=0
$$

Finally, the pressure-displacement law can be expressed in $(\xi, \eta)$-space as

$$
P_{1}=F_{2} \cos \phi-\frac{\sin \phi}{3 W e} \frac{\partial \xi}{\partial x_{1}}\left(\frac{\partial^{2} F_{2}}{\partial \xi^{2}} \frac{\partial \xi}{\partial x_{1}}+\frac{\partial F_{2}}{\partial \xi} \frac{\partial^{2} \xi}{\partial x_{1} \partial \xi}\right) .
$$

For computational purposes, we cannot set the upper boundary at $\eta=1$, as the boundary conditions on the outer edge of the viscous sublayer contain $y_{1}(\xi, \eta)$. Therefore, the outer-edge boundary conditions are evaluated at a cutoff value $\eta=\eta_{e}$. Note that in this case we have compression of the grid provided via the transform (4.6), and $\eta_{e}$ can be set to a sufficiently large value so that second-order corrections to boundary conditions become unnecessary.

The boundary conditions on the ice surface and the outer edge of the viscous sublayer are given by

$$
\left.\begin{array}{c}
u_{1}=0, \quad v_{1}=0, \quad T_{1}=0, \\
a \frac{\partial H_{1}}{\partial t_{1}}=-\frac{\partial T_{1}}{\partial \eta} \frac{\partial \eta}{\partial y_{1}}-\lambda_{i l} \frac{T_{w}}{H_{1}+H_{0}},
\end{array}\right\}
$$

at $\eta=0$ and

$$
v_{1}=3 y_{1} \frac{\partial F_{2}}{\partial x_{1}}, \quad u_{1}=3 y_{1}(\xi, \eta)-3 F_{2}, \quad T_{1}=y_{1}(\xi, \eta)-F_{2}, \text { at } \eta=\eta_{e},
$$


respectively. On the left boundary we assume undisturbed flow

$$
\left.\begin{array}{rl}
u_{1} & =3 y_{1}(\xi, \eta), \\
v_{1} & =0 \\
T_{1} & =-y_{1}(\xi, \eta),
\end{array}\right\} \xi \rightarrow-1
$$

and on the right boundary the required quantities are obtained by linear extrapolation from the computational domain.

The algorithm applied to obtain the numerical solution can be described as follows:

(a) Consider the ice surface shape $H_{1}$ to be fixed at some time step.

(b) For this fixed ice surface shape, we perform $u_{1}-v_{1}-F_{2}$ global marching iterations while the appropriate derivative in pseudo-time (used to construct the iterations) exceeds computational precision.

(c) Once the momentum equations have converged, we perform global marching iterations for the temperature equation for fixed ice- and free- surface shapes and known velocity field.

(d) As a result of steps $(a)-(c)$, we obtain the solution for the temperature field corresponding to the fixed ice shape at a given time step. Then the Stefan condition is used in order to find the ice surface shape at the next time step.

The momentum equations were discretized using a second order in space and first order in pseudo-time finite-difference scheme. The iteration function $Q_{c}$ was set to $\left(u_{1}^{2}, u_{1} T_{1}\right)^{T}$, which is equivalent to the solution of unsteady equations with a local non-uniform time step defined by $\Delta \tau^{l}=\Delta \tau / u_{1}$. This approach proved to increase the speed of convergence towards a steady-state solution in comparison to computations with a constant time step. A detailed description of the numerical scheme can be found in Shapiro (2004).

In the computations presented in this section we have used fixed values for the angle of incline (0.001) and for the Weber number (1000). In order to compare nonlinear results with linear results for the same configuration, we note that if media properties, the angle of incline and the Weber number are all fixed, then the only free parameter left in the linear problem is the initial ice height $H_{0}$.

Consider first flow regimes with strong linear instability. The analytic results in the previous section indicate that the growth rate increases with the initial ice height and that the neutral stability curve tends to a limiting curve as $H_{0} \rightarrow \infty$. In order to investigate the effects of nonlinearity in a highly linearly unstable flow we have chosen $H_{0}=1000$, based on the behaviour of the linear stability neutral curve. The initial perturbation of the ice surface was taken to be of the form $H_{a} \mathrm{e}^{-x_{1}^{2}}$ with the amplitude $H_{a}=0.1$.

The nonlinear disturbance evolves as a wave packet. Compared to the linear regime, the nonlinear solution has a similar phase speed at small times. However, the growth rate is smaller than that of the linear wave packet (figure 8). On a longer time scale, it becomes noticeable that the nonlinear solution does not possess the strong dispersion characteristics of the linear disturbance, and that the propagation speed of the first peak is higher in the nonlinear case. Figure 9 shows the evolution of the ice surface for linear and nonlinear problems at large times. Again, comparison between linear and nonlinear solutions demonstrates lower growth rate due to nonlinearity and faster upstream propagation of the nonlinear disturbance. Finally at 60 time units, the linear solution represents a fully developed wave packet, while in the nonlinear solution only one secondary peak is observed (figure 10). The free-surface shape follows distortions in the ice surface but with slightly larger amplitude (figure 11). 

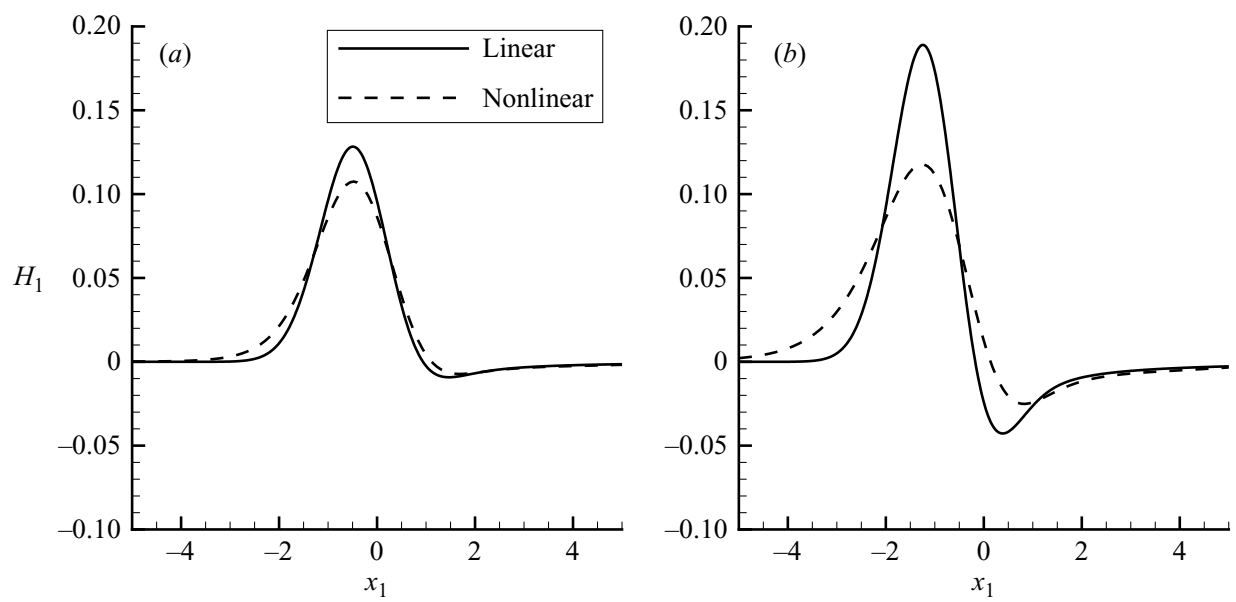

FiguRE 8. Linear and nonlinear ice surface, initial stages: $(a) t_{1}=4,(b) t_{1}=10$.
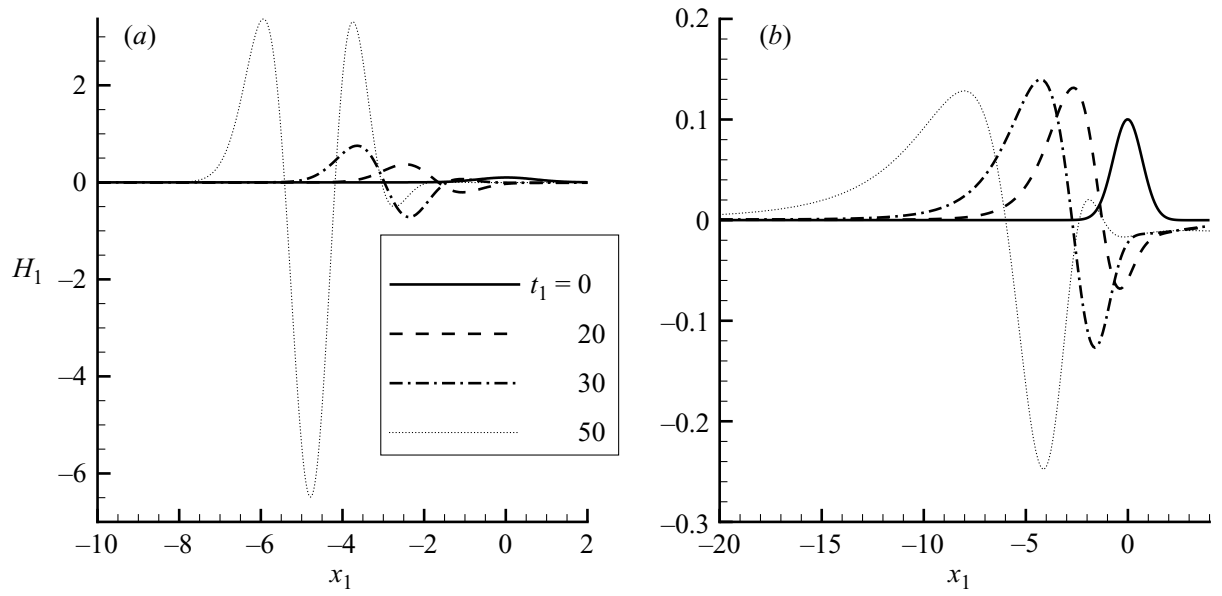

Figure 9. (a) Linear and (b) nonlinear ice surface evolution, large time scale.
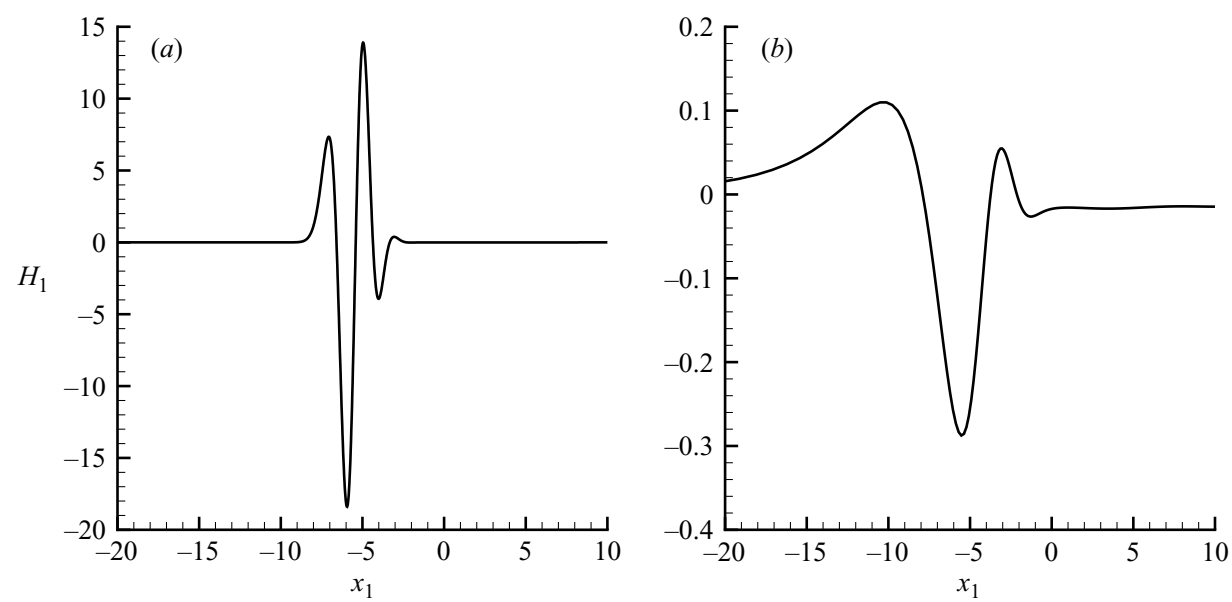

FIGURE 10. (a) Linear and (b) nonlinear ice surface shape, $t_{1}=60$. 

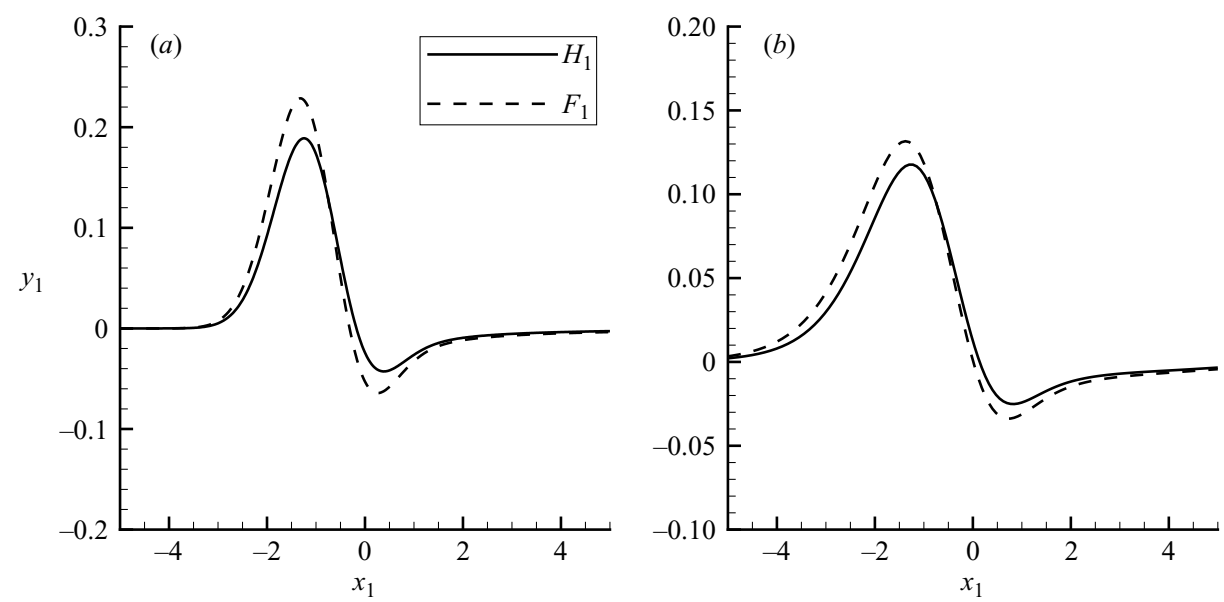

FIgURE 11. Comparison of free surface and ice shapes, $t_{1}=10:(a)$ linear, $(b)$ nonlinear.
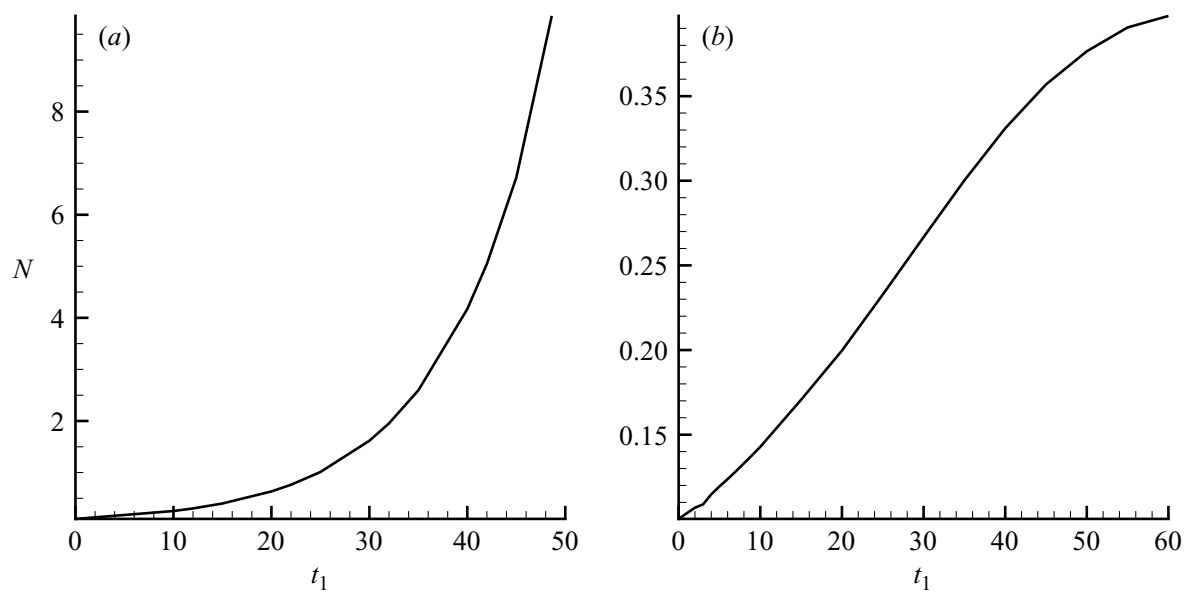

FIGURE 12. Ice-surface amplitude evolution: $(a)$ linear, $(b)$ nonlinear.

Owing to the slow evolution of the nonlinear wave packet and its rapid upstream propagation, it was not possible to follow the solution development on very large time scales as the numerical grids that could be used proved insufficient to resolve the solution far upstream. However a certain tendency of the limiting behaviour can be seen if we consider a suitable norm of the solution and plot it as a function of time. In order to do this, we have chosen to use the following norm:

$$
N=\|H\|=\max _{i=1, N x} H_{i}-\min _{i=1, N x} H_{i},
$$

where $N_{x}$ denotes the total number of grid nodes in the $x_{1}$ direction. This choice was motivated by the fact that the absolute-value norm will not represent the total variation of the solution. The comparison between the norm of linear and nonlinear solutions is shown in figure 12 for the ice-surface shape. The norm of the linear solution grows exponentially as expected; however, the norm of the nonlinear wave packet exhibits linear growth initially and then tends to stabilize. 


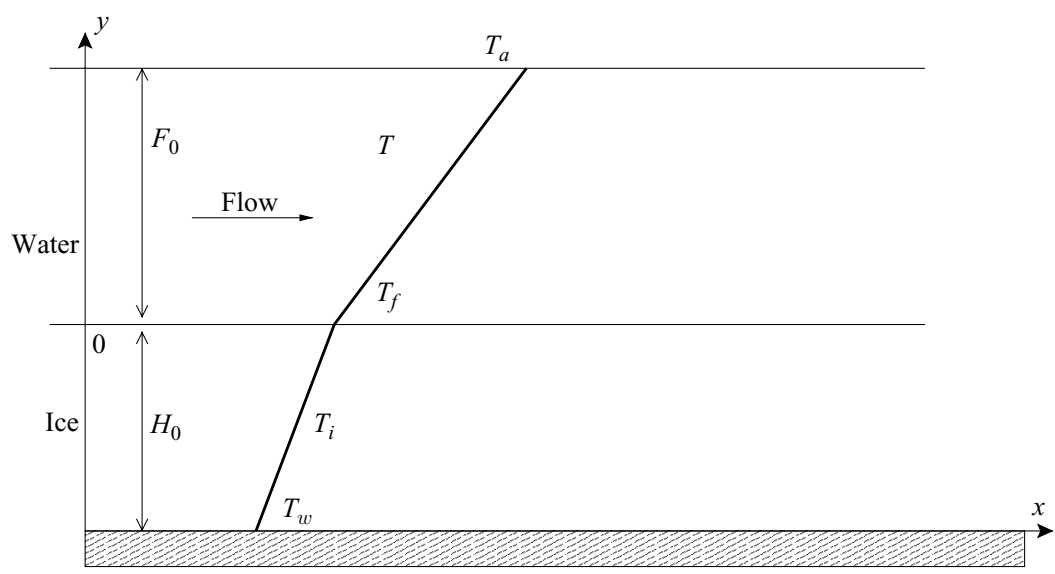

FIGURE 13. Liquid/solid interface instability mechanism, initial state.

\section{A mechanism for upstream propagation of icing instability}

Based on the observed behaviour of icing instability, it is possible to propose a simple physical explanation of the upstream propagation of growing distortions in the ice surface. Moreover, one can derive such an explanation based solely on an interaction between the free-surface disturbances and perturbations in the shape of the ice layer. Consider a two-dimensional liquid film flow over a solid phase under the following assumptions:

(a) The time scale of the solidification process is much larger than that of the liquid flow.

(b) The initial distribution of temperature is linear and both the solid/liquid interface and the free surface are flat initially.

(c) The temperature is constant on the lower boundary of the solid phase $T=T_{w}$ and also at the solid/liquid interface $T=T_{f}$ and on the free surface $T=T_{a}$.

In the equilibrium state, the heat flux balance on the liquid/solid interface can be written as

$$
\lambda_{i} \frac{\partial T_{i}}{\partial y}=\lambda_{l} \frac{\partial T}{\partial y} .
$$

Since the unperturbed temperature profile is linear, the temperature gradients expressed in terms of the boundary values and initial thicknesses of the solid and liquid layers, $H_{0}$ and $F_{0}$, are given by

$$
\frac{\partial T_{i}}{\partial y}=\frac{T_{f}-T_{w}}{H_{0}}
$$

and

$$
\frac{\partial T}{\partial y}=\frac{T_{a}-T_{f}}{F_{0}}
$$

respectively. Consider a localized small perturbation of the ice surface, $h(x)$, small enough for the temperature profiles to remain linear at leading order. This assumption can be relevant, for example, to long-wave disturbances. Since we assume that the time scale of the solid surface growth is much larger than the time scale of the flow, the flow in the liquid phase can be treated as quasi-steady so that the flow field can be determined initially for a fixed shape of the solid surface $h(x)$. This will give us the perturbation of the free surface $f(x)$. The problem of a liquid film flow 
over an obstacle is thoroughly investigated and it is known that perturbations in the free surface are able to propagate upstream of the obstacle (see, for example, Bowles 1995). This mechanism of upstream influence has no immediate relation to hydrodynamic instabilities in liquid films. Let us consider a point $x_{0}$ located in front of the liquid/solid interface perturbation. If the solid/liquid perturbation is small, and the temperature distribution can still be considered linear, then at the ice surface the heat flux is no longer balanced, specifically

$$
\lambda_{i} \frac{T_{f}-T_{w}}{H_{0}}>\lambda_{l} \frac{T_{a}-T_{f}}{F_{0}+f\left(x_{0}\right)} .
$$

The heat flux from the liquid side decreases as the thickness of the liquid layer increases and therefore the liquid/solid interface has to lift up in order to compensate the heatflux imbalance. This in turn causes the ice perturbation to propagate upstream.

\section{Conclusions}

In this paper we develop an asymptotic theory for a boundary-layer-type flow in a liquid film at large Froude numbers. We consider linear and nonlinear stability of the flow with a half-Poiseuille velocity profile down an inclined plane covered with an ice layer. This leads to the description of a new mode of upstream propagating instability arising because of the presence of the ice layer.

In the linear approximation for the viscous sublayer of the asymptotic double-deck theory, we derive an exact solution in terms of the Airy functions in the threedimensional and two-dimensional cases for $\operatorname{Pr}=1$. In the general case with $\operatorname{Pr} \neq 1$, we provide a numerical solution for three-dimensional and two-dimensional linear disturbances. Using the numerical solution of the linear problem, we show that our closed-form solution is indeed applicable to the flow with $\operatorname{Pr}=13.47$ (as is the case for water near freezing temperature), provided the time is rescaled accordingly. This suggests that the thermal boundary layer affects the speed of propagation of small disturbances but has no significant impact on the shape of disturbances. In the threedimensional case we found that the flow can become unstable to oblique waves before it becomes unstable to two-dimensional perturbations provided the initial thickness of the undisturbed ice layer is small. This can provide an explanation for the initial growth of ice-surface perturbations at an angle to the flow direction as observed in the experiments by Streitz \& Ettema (2002).

For nonlinear double-deck equations, we develop a novel global-marching-type numerical scheme and use it to study the evolution of nonlinear two-dimensional disturbances. The computed solution demonstrates that nonlinearity tends to weaken the dispersive properties of the individual wave components with a decrease in the growth rates and an increase in the speed of upstream propagation.

The mode of the icing instability we discover in the flows governed by the largeFroude-number double-deck theory does not require cooling below the freezing point at the free surface (as is often the case in wind tunnel investigations of airfoil icing). This instability is also distinct from the short-scale instability of supercooled liquids resolved on the shortest scales by the freezing temperature modification via the GibbsThomson effect. We also show that the icing instability in a gravity-driven liquid layer is different from the flow-induced morphological instabilities of the solidification front, the latter also being able to propagate upstream under certain conditions (see, for example Brattkus \& Davis 1988; Forth \& Wheeler 1989; Batchelor et al. 2000). The icing instability in this study is due to an interaction between the free surface 
of the liquid layer and the liquid/solid interface via heat fluxes at the boundaries combined with the pressure-induced upstream influence in the liquid phase.

The asymptotic theory in this paper inevitably has its limits, since we use approximations to the equations of motion formally valid when both the Reynolds and Froude numbers are large. The formal domain of its validity is discussed in detail in $\S 2$. However, a linear stability analysis reported in a recent paper by Shapiro \& Timoshin (2006) confirms the presence of the icing instability mode in flows with finite values of the control parameters.

Useful discussion and helpful comments of the referees are gratefully acknowledged.

\section{REFERENCES}

Abramowitz, M. \& Stegun, I. A. 1972 Handbook of Mathematical Functions (with Formulas, Graphs, and Mathematical Tables). Dover.

Alexiades, V. \& Solomon, A. D 1993 Mathematical modeling of Melting and Freezing Processes. Hemisphere.

Batchelor, G. K., Moffatt, H. K. \& Worster, M. G. 2000 Perspectives in Fluid Dynamics: A Collective Introduction to Current Reasearch. Cambridge University Press.

Bowles, R. I. 1995 Upstream influence and the form of standing hydraulic jumps in liquid-layer flows on favourable slopes. J. Fluid. Mech. 284, 63.

BRATtKus, K. \& Davis, S. H. 1988 Flow-induced morphological instabilities: stagnation-point flows. J. Cryst. Growth 89, 423.

Briggs, R. J. 1964 Electron-Stream Interaction with Plasmas. MIT Press.

Cassel, K. W., Ruban, A. I. \& Walker, J. D. A. 1995 An instability in supersonic boundary-layer flow over a compression ramp. J. Fluid Mech. 300, 265.

Chung, T. J. 2002 Computational Fluid Dynamics. Cambridge University Press.

DAvis, S. H. 1990 Hydrodynamic interactions in directional solidification. J. Fluid. Mech. 212, 241.

Forth, S. A. \& WheEler, A. A. 1989 Hydrodynamic and morphological stability of unidirectional solidification of a freezing binary alloy: a simple model. J. Fluid Mech. 202, 339.

GaJjar, J. S. B. 1987 Fully developed free surface flows - liquid layer flow over a convex corner. Computers Fluids 15, 337-360.

Gilpin, R. R. 1979 The morphology of ice structure in a pipe at or near transient Reynolds numbers. Heat Transfer - San Diego, AIChE Symposium Series 189, vol. 75 , p. 89.

GiLPIN, R. R. 1981 Ice formation in a pipe containing flows in the transition and turbulent regimes. J. Heat Transfer 103, 363.

Glicksman, M. E., Coriell, S. R. \& McFadden, G. B. 1986 Interaction of flows with the crystalmelt interface. Annu. Rev. Fluid Mech. 18, 307.

Hooper, A. P. \& Boyd, W. G. C. 1983 Shear-flow instability at the interface between two shearing fluids. J. Fluid Mech. 128, 507.

IsaAcson, E. \& Keller, H. B. 1966 Analysis of Numerical Methods. Dover.

Kikuchi, Y., Shigemasa, Y., Oe, A. \& Ogata, T. 1986 Steady-state freezing of liquids in laminar flow between two parallel plates. J. Nucl. Sci. Technol. 23, 979.

LEE, J. S. 1993 Freezing problems in pipe flows. PhD thesis, Iowa State University.

RothmaYer, A. P. \& Tsao, J. C. 1998 A mechanism for ice roughness formation on an airfoil leading edge, contributing to glaze ice accretion. AIAA Paper 98-0485.

Rothmayer, A. P. \& TsaO, J. C. 2000 Triple-deck simulation of surface glaze ice accretion. AIAA Paper 2000-0234.

Seki, N., Fukusako, S. \& Younan, G. W. 1984 Ice-formation phenomena for water flow between two cooled parallel plates. J. Heat Transfer 106, 498.

ShapIRo, E. 2004 Instabilities in multiphase and icing flows. PhD thesis, University of London.

Shapiro, E. \& Timoshin, S. 2006 Linear stability of ice growth under a gravity-driven water film. Phys. Fluids 18, 074106.

Streitz, J. T. \& Ettema, R. 2002 Observations from an aufeis windtunnel. Cold Regions Sci. Technol. 34(2), 85 . 
Sychev, V. V., Ruban, A. I., Sychev, V. V. \& Korolev, G. L. 2000 Asymptotic Theory of Separated Flows. Cambridge University Press.

Timoshin, S. N. 1997 Instabilities in a high-reynolds-number boundary layer on a film-coated surface. J. Fluid Mech. 353, 163.

Timoshin, S. N. \& Hooper, A. P. 2000 Mode coalescence in two-fluid boundary-layer stability problem. Phys. Fluids 12, 1969.

Vargas, M. \& Reshotko, E. 1998 Physical mechanisms of glaze ice scallop formations on swept wings. AIAA Paper 98-0491.

YaO, L. S. \& Prusa, J. 1989 Melting and freezing. Adv. Heat Transfer 19, 1. 